\title{
Alleviation of Cadmium Toxicity in Zea Mays L. through Up-Regulation of Antioxidant Defense System and Organic Osmolytes under Supplemental Calcium
}

Muhammad Kaleem ( $\sim$ kaleemakmal5798@gmail.com )

University of Agriculture Faisalabad https://orcid.org/0000-0003-3237-4886

Iqbal Hussain

Government College University Faisalabad

Mansoor Hameed

University of Agriculture Faisalabad

Muhammad Sajid Aqeel Ahmad

University of Agriculture Faisalabad

Anam Mehmood

Government College University Faisalabad

Muhammad Faisal Maqsood

University of Agriculture Faisalabad

Ummar lqbal

University of Agriculture Faisalabad

\section{Zunaira Anwar}

University of Agriculture Faisalabad

Syed Mohsan Raza Shah

University of Agriculture Faisalabad

Waseem Ashfaq

The University of Melbourne

\section{Research Article}

Keywords: Antioxidants, cadmium, calcium, maize, oxidative stress, reactive oxygen species

Posted Date: July 19th, 2021

DOl: https://doi.org/10.21203/rs.3.rs-611406/v1

License: (a) This work is licensed under a Creative Commons Attribution 4.0 International License.

Read Full License 
Version of Record: A version of this preprint was published at PLOS ONE on June 22nd, 2022. See the published version at https://doi.org/10.1371/journal.pone.0269162. 


\section{Abstract}

Calcium (Ca) is a macronutrient and work as a modulator to mitigate oxidative stress induced by heavy metals. Present work was conducted to elucidate the role of $\mathrm{Ca}$ in modulating growth, physio-biochemical traits, and cellular antioxidant defense system in Zea mays L. seedlings under Cd stress. The experiment was designed in a complete randomized design with two levels of $\mathrm{Cd}(0$ and $150 \mu \mathrm{M})$ and six levels of $\mathrm{Ca}$ $(0,0.5,1,2.5,5$ and $10 \mathrm{mM})$. Maize seedlings exposed to $\mathrm{Cd}$ at $150 \mu \mathrm{M}$ concentration showed a notable decrease in growth, biomass, anthocyanins, chlorophylls, and antioxidant enzymes activities. Higher level of $\mathrm{Cd}(150 \mu \mathrm{m})$ also caused an upsurge in oxidative damage observed as higher electrolyte leakage (increased membrane permeability), $\mathrm{H}_{2} \mathrm{O}_{2}$ production and MDA accumulation. Supplementation of $\mathrm{Ca}$ notably improved growth traits, photosynthetic pigments, cellular antioxidants (APX, POD and ascorbic acid), anthocyanins and level of osmolytes. The significant improvement in the osmolytes (proteins and amino acids), and enzymatic antioxidative defense system enhanced the membrane stability and mitigated the damaging effects of $\mathrm{Cd}$. The present results concluded that exogenously applied Ca can potentially improve growth by regulating antioxidants and enable maize plants to withstand the $\mathrm{Cd}$ toxicity.

\section{Introduction}

While growing in natural environments, plants are exposed to various environmental stresses that limit yield and productivity (Huybrechts et al. 2019). Heavy metal pollution is spreading in cultivated lands and is causing severe environmental hazards to crop plants, human health and ecosystems (Liu et al. 2014). Cd is regarded as the most toxic heavy metal, typically when present in agricultural lands due to its higher mobility and toxicity (Goix et al. 2014). Plants can readily absorb Cd through roots directly from the soil along with essential nutrients (Nogueirol et al. 2016). Like other heavy metals, Cd causes structural changes in plants and adversely affects the morphological, physiological, and biochemical mechanisms which eventually lead to loss of agricultural productivity (Kováčik et al. 2014). Cd is typically nonessential for agricultural crops as no known role is ascribed for $\mathrm{Cd}$ in growth and development of crop plants (Huybrechts et al. 2019). Therefore, Cd even in minor concentrations disturb the photosynthesis, change the ultrastructure of the chloroplast, increase lipid peroxidation and enhance production of ROS that leads to oxidative damage (Gallego et al. 2012; Dourado et al. 2015; Gratão et al. 2015). Another direct effect of high $\mathrm{Cd}$ is production of excessive ROS $\left(\mathrm{H}_{2} \mathrm{O}_{2}, \mathrm{OH}^{-}, \mathrm{O}_{2} \cdot{ }^{-},{ }^{1} \mathrm{O}_{2}\right)$ resulting in lipids peroxidation which ultimately reduces plant growth (Maleki et al. 2017).

Many defensive mechanisms are induced in plants to counteract $\mathrm{Cd}$ toxicity mainly by hyper production of antioxidants (non-enzymatic or enzymatic) to control heavily produced ROS (Kováčik et al. 2014; Asgher et al. 2014). These enzymatic antioxidants (like peroxidase, superoxide dismutase, ascorbate peroxidase, and catalases), and non-enzymatic anti-oxidants (such as a-tocopherol and glutathione) have been reported to successfully mitigate Cd-induced oxidative damage in many crop plants (Mishra et al. 2014; Maleki et al. 2017). Other reports show that heavy metals may results in hyper-accumulation of proteins as an effective strategy to mitigate Cd induced toxicity (El-Beltagi and Mohamed 2013). 
Calcium $\left(\mathrm{Ca}^{2+}\right)$ is an essential macromolecule and divalent cation that performs an imperative role in membrane permeability, metabolism and signal transduction (El-Beltagi and Mohamed 2013; Vigani and Costa 2019; Malik et al. 2020). It is a central regulator in the physio-chemical process and regulates plant growth (Siddiqui et al. 2012). Exogenously applied Ca alleviates oxidative stress by chelating with target proteins (for instance calcium-binding proteins) and activating the antioxidant enzymes (Song et al. 2009; Grzybowska 2018). Though, the induction of antioxidative defense system by $\mathrm{Ca}$ is not yet elucidated sufficiently, however, some reports support that $\mathrm{Ca}$ is involved in the modulation of genes for antioxidant enzymes (Niu and Liao 2016). Ca mitigates Cd toxicity in plants by modifications in morphological and physiological process (Siddiqui et al. 2012; Ahmad et al. 2015). For example, Ca maintains permeability of membranes by a reduction in peroxidation of lipids and solute leakage which ultimately reduces oxidative stress caused by Cd stress (Lwalaba et al. 2017). In other reports, Ca improved growth and photosynthesis by restricting Cd translocation and accumulation, scavenging ROS, enhancing antioxidant level, and maintaining Ca-dependent signal transduction (Srivastava et al. 2015; Huang et al. 2017). Still, the ameliorative role of Ca to alleviate heavy metals toxicity remains inconclusive and therefore it is imperative to investigate its specific roles and associated mechanisms in improving growth of Zea mays L. seedlings.

Maize is a valuable cereal crop and provides food for humans as well as fodder for the livestock. It contributes to $36 \%$ (782 Mt) in global grain production (Sah et al. 2020). Maize seeds are enriched with energy as $100 \mathrm{~g}$ seeds contain 365 kilocalories of energy (Nuss and Tanumihardjo 2010). Among worldwide production, $70-80 \%$ of maize is used as food and was ranked third in Pakistan for consumption after wheat and rice. Pakistan was ranked 18th in the production with 6130 thousand tons maize produced annually that was cultivated at 1334 thousand hectares (Rani et al. 2015). The requirement for the maize production has significantly increased recently due to excessive usage in the wet milling industry as well as food for poultry (Boomsma and Vyn 2008). This needs not only to increase the cultivation area but also the exploration of new promising techniques to increase crop survival and yield under stressful environments like in soils contaminated with heavy metals (Khan 2020).

Cadmium contamination is gradually increasing in soils and is causing significant crop losses. Therefore, there is a dire need to devise new strategies to combat the problems associated with Cd toxicity. Considering many critical roles played by $\mathrm{Ca}$ in plant growth and metabolism, it was hypothesized that Ca supplementation should effectively ameliorate the adverse effects of $\mathrm{Cd}$ imposed on germinating seeds of maize. The research questions included to probe into the toxic effects of $\mathrm{Cd}$ on the growth, physio-biochemical characteristics and to what extent supplemental $\mathrm{Ca}$ can alleviate $\mathrm{Cd}$ toxicity in maize. These findings would contribute to explore the mechanism and recommendation of the best levels of $\mathrm{Ca}$ in ameliorating $\mathrm{Cd}$ toxicity in maize.

\section{Materials And Method}

Plant materials and layout 
Maize seeds (Sahiwal-2002) were procured from Maize and Millets Research Institute (MMRI), Yousaf Wala Sahiwal, Pakistan. Seeds were immersed in $30 \%(\mathrm{v} / \mathrm{v}) \mathrm{H}_{2} \mathrm{O}_{2}$ for 5 min. for sterilization, washed with deionized water for $24 \mathrm{~h}$ and dried. Seeds were placed in Petri dishes lined with double layer of What-man \# 02 filter papers. The surface of each filter paper was moistened with $15 \mathrm{~mL}$ of $\mathrm{H}_{2} \mathrm{O}$ and kept in dark condition $25 \pm 2{ }^{\circ} \mathrm{C}$ for a $48 \mathrm{~h}$. After germinations, six seeds were planted in plastic pots (depth; $40 \mathrm{~cm}$ and diameter; $35 \mathrm{~cm}$ ) in sterilized sand with a particular particle size $0.25 \mathrm{~mm}$. The sand was soaked for $24 \mathrm{~h}$ in $30 \%(\mathrm{v} / \mathrm{v}) \mathrm{HCl}$ solution to remove all cations and anions and then thoroughly rinsed with deionized water three time (with $24 \mathrm{~h}$ soaking). The pots were treated with 0 and $150 \mu \mathrm{m} \mathrm{Cd}^{2+}$ applied as $\mathrm{CdCl}_{2}$ and $0,0.5,1,2.5,5$ and $10 \mathrm{mM} \mathrm{Ca}^{2+}$ levels using $\mathrm{Ca}\left(\mathrm{NO}_{3}\right)_{2}$ as source. The $1 / 2$-strength Hoagland's (Hoagland 1938) nutrient solution was applied to the seedlings throughout the experimentation. The Hoagland solution was applied to the plants to saturate the sand at 3 days interval with draining any past solution left in sand. All pots were arranged as CRD with 3 replications under controlled glasshouse environment with day/night temperature of $24 \pm 4^{\circ} \mathrm{C} / 14 \pm 2^{\circ} \mathrm{C}$ and $\mathrm{RH} 58-60 \%$ (Rady et al. 2019).

\section{Plant sampling and measurements}

Plants material was sampled at the seedling stage to determine plant growth attributes, physiobiochemical traits, ROS, and enzymes of antioxidants defense system. Harvested seedlings were washed with distilled water and growth attributes were recoded. Leaf samples of maize seedlings were frozen at $-80^{\circ} \mathrm{C}$ for physio-biochemical traits and antioxidants. Sampled seedlings were dried in oven at $70{ }^{\circ} \mathrm{C}$ to achieve a constant dry weight for determination of root (RDW) and shoot (SDW) dry weight.

\section{Growth attributes}

The shoot length (SL) of plants from each treatment was measured from sand level to the topmost leaf of the plant. The roots of seedlings were carefully removed from the sand for recording root length (RL). Root (RFW) and shoot fresh weight (SFW) of seedlings were measured immediately after excision. The leaf area $(\mathrm{LA})$ was estimated by measuring length $\times$ width $\times 0.68$.

\section{Physiological attributes}

\section{Chlorophyll contents}

Chlorophyll contents were assessed as described by Arnon (1949) and carotenoids following the method of Davis (1976). For the appraisal of chlorophyll contents, $0.1 \mathrm{~g}$ of leaf sample was grounded in $5 \mathrm{~mL}$ of acetone ( $80 \%)$. Extract was filtered through a Whatman \# 02 filter paper (GE Healthcare, UK) and absorbance was recorded through a spectrophotometer (Hitachi U-2910, Tokyo, Japan) at 645, 663, and $480 \mathrm{~nm}$. The values of photosynthetic pigments were calculated by using the following formula. 
Chl. $a(\mathrm{mg} / \mathrm{g}$ of leaf FW $)=[12.7(\mathrm{OD} 663)-2.69(\mathrm{OD} 645)] \times \mathrm{V} / 1000 \times \mathrm{W}$

Chl. $b(\mathrm{mg} / \mathrm{g}$ of leaf FW $)=[22.9(\mathrm{OD} 645)-4.68(\mathrm{OD} 663)] \times \mathrm{V} / 1000 \times \mathrm{W}$

Total Chl. $(\mathrm{mg} / \mathrm{g}$ of leaf FW $)=[20.2(\mathrm{OD} 645)+8.02(\mathrm{OD} 663)] \times \mathrm{V} / 1000 \times \mathrm{W}$

Carotenoids $(\mathrm{g} / \mathrm{ml}$ of $\mathrm{FW})=\{[(\mathrm{OD} 480)+0.114(\mathrm{OD} 663)-0.638(\mathrm{OD} 645)] / 2500\}$

Here, $V$ characterizes the volume of acetone and (FW) showed the leaf fresh weight.

\section{Determination of relative membrane permeability}

The fresh leaf samples were collected and washed thoroughly with 4 changes of water to eradicate any adhered electrolytes on the surface. The leaves were cut into small discs with a borer and placed in the small glass test tube containing deionized water $(10 \mathrm{~mL})$, The $\mathrm{EC}_{0}$ was measured by the help of Cond/Salinity meter (TPS AQUA-CPA). The test tubes were incubated for $24 \mathrm{~h}$ at $4{ }^{\circ} \mathrm{C}$ and $\mathrm{EC}_{1}$ was measured. The tubes were then wrapped with aluminum foil, autoclaved for $10 \mathrm{~min}$. at $100 \mathrm{kPa}$ and $\left(\mathrm{EC}_{2}\right)$ was recorded. The ratio of \% ion leakage was computed as designated by Yang et al. (1996).

\section{$\mathrm{RMP}(\%)=\left(\mathrm{EC}_{1}-\mathrm{EC}_{0}\right) /\left(\mathrm{EC}_{2}-\mathrm{EC}_{0}\right) \times 100$}

\section{Assessment of biochemical traits}

\section{Anthocyanin contents}

Anthocyanin content was appraised according to the method of Giusti and Wrolstad (2001). The $0.1 \mathrm{~g}$ of leaf was pulverized in trichloroacetic acid (TCA) by using pestle and mortar. The homogenized material was transferred to test tubes and shifted to water bath at $80^{\circ} \mathrm{C}$ for $20 \mathrm{~min}$. Homogenized material was centrifuged at 12,000 $\mathrm{xg}$ for $10 \mathrm{~min}$. in the absorbance was noted at 516 and $700 \mathrm{~nm}$ using a spectrophotometer (Hitachi U-2910, Tokyo, Japan). Acetone was run as blank and amount of monomeric anthocyanin contents was calculated as follows.

Chl. $a(\mathrm{mg} / \mathrm{g}$ of leaf FW $)=[12.7(\mathrm{OD} 663)-2.69(\mathrm{OD} 645)] \times \mathrm{V} / 1000 \times \mathrm{W}$

Chl. $b(\mathrm{mg} / \mathrm{g}$ of leaf FW $)=[22.9(\mathrm{OD} 645)-4.68(\mathrm{OD} 663)] \times \mathrm{V} / 1000 \times \mathrm{W}$

Total Chl. $(\mathrm{mg} / \mathrm{g}$ of leaf $\mathrm{FW})=[20.2(\mathrm{OD} 645)+8.02(\mathrm{OD} 663)] \mathrm{x} \mathrm{V} / 1000 \mathrm{x}$ W

Carotenoids $(\mathrm{g} / \mathrm{ml}$ of $\mathrm{FW})=\{[(\mathrm{OD} 480)+0.114(\mathrm{OD} 663)-0.638(\mathrm{OD} 645)] / 2500\}$ 
Here, $A=(A 510-A 700) M W=449.2$ and $\varepsilon=26900$ [ $\varepsilon$ is the molar absorptivity measured the amount of cyanidin-3-glucoside pigment and DF is dilution factor].

\section{Oxidative stress markers (MDA and $\mathrm{H}_{2} \mathrm{O}_{2}$ )}

Lipids peroxidation (LPX) was quantified by means of malondialdehyde (MDA contents) by following Heath and Packer (1968). LPX content was determined by the reaction of thiobarbituric acid-TCA with trichloroacetic acid-TCA. The $0.25 \mathrm{~g}$ leaf sample was grinded in $500 \mu \mathrm{L}$ of TCA $(0.1 \%)$ and then centrifuged at 15,000 $\mathrm{xg}$. An aliquot $(1 \mathrm{~mL})$ was taken and mixed with $2 \mathrm{~mL}$ of $0.5 \%$ of TBA and $20 \%$ TCA. Test tubes containing reactants were incubated at $85^{\circ} \mathrm{C}$ for $20 \mathrm{~min}$. and reaction was terminated in an icebox. Absorption was recorded at 532 and $600 \mathrm{~nm}$ by spectrophotometer (Hitachi U2910, Tokyo, Japan). All absorption ODs (at 532nm) were subtracted from $600 \mathrm{~nm}$. LPX concentration was calculated by using $155 \mathrm{mM} \mathrm{cm}^{-1}$ as an extinction coefficient.

Amount of $\mathrm{H}_{2} \mathrm{O}_{2}$ was quantified by measuring the oxidation of ferrous ions medicated by peroxidase and ferric ions react with the xylenol (Bellincampi et al. 2000). Leaf sample $0.5 \mathrm{~g}$ was grounded in $5 \mathrm{~mL}$ of 10 $\mathrm{mM}$ sodium phosphate buffer (SPB). Centrifugation of homogenized material was done at 15,000 xg. A 2 $\mathrm{mL}$ of aliquot was reacted with the assay reagent containing $200 \mathrm{mM}$ sorbitol, $200 \mu \mathrm{M}$ xylenol, $50 \mathrm{~mm}$ $\mathrm{H}_{2} \mathrm{SO}_{4}$, and $500 \mu \mathrm{M}$ ammonium ferrous sulphate. The reactant material was incubated at $24^{\circ} \mathrm{C}$ for a $1 \mathrm{~h}$ and absorption was recorded at $560 \mathrm{~nm}$ by using a spectrophotometer (Hitachi U-2910, Tokyo, Japan).

\section{Cellular antioxidants (APX and POD)}

The maize seedlings' shoot were grounded in liquid nitrogen and extracted with $1 \mathrm{mM} \mathrm{L}^{-1}$ of $5 \%$ polyvinylpyrrolidone, and, sodium phosphate buffer (SPB) having $\mathrm{pH}$ 7.8. Extracted material was centrifuged at $15,000 \mathrm{xg}$. Enzyme crude extract was stored at $4{ }^{\circ} \mathrm{C}$ for $36 \mathrm{~h}$ till analysis.

\section{Ascorbate peroxidase activity (APX)}

Activity of APX was quantified by oxidation of ascorbate (Chen and Asada 1989). Reaction was started by adding $10 \mu \mathrm{L}$ of crude enzyme extract to $2 \mathrm{~mL}$ of assay reagent $\left(30 \% \mathrm{H}_{2} \mathrm{O}_{2}, 0.5 \mathrm{mM} \mathrm{C}_{6} \mathrm{H}_{8} \mathrm{O}_{6}\right.$, and sodium phosphate buffer (SPB) having $\mathrm{pH}$ 7.2,). After $30 \mathrm{~s}$ of reaction initiation, a shift in absorption was noted at $290 \mathrm{~nm}$ for $4 \mathrm{~min}$. on a spectrophotometer (Hitachi U-2910, Tokyo, Japan). Activity of enzyme was estimated through extinction coefficient $\left(2.8 \mathrm{mM} \mathrm{cm}^{-1}\right)$, while the specific activity of the enzyme was calculated on the basis of protein contents and expressed as an $\mathrm{mg}^{-1} \mathrm{~min}^{-1} \mathrm{FW}$.

\section{Peroxidase (POD) activity}

POD activity was appraised spectrophotometrically by using the method of Goliber (1989) based on oxidized of guaiacol in the presence of $\mathrm{H}_{2} \mathrm{O}_{2}$ and expressed as a Units $\mathrm{mg}^{-1}$ proteins. A $20 \mu \mathrm{L}$ of the enzyme extract was added to assay reagent ( $20 \mathrm{mM}$ guaiacol, $10 \mathrm{mM} \mathrm{H}_{2} \mathrm{O}_{2}$, and $0.1 \mathrm{M}$ phosphate buffer) and volume was maintained up to $3 \mathrm{~mL}$. Enzyme activity was measured at $460 \mathrm{~nm}$ after $60 \mathrm{~s}$ interval 
through a spectrophotometer (Hitachi U-2910, Tokyo, Japan). Enzyme specific activity was expressed on the base of proteins.

\section{Ascorbic acid}

Ascorbic acid was determined as described by Nino and Shah (1986). Plant tissues (100 mg) were pulverized in thiobarbituric acid (TCA) and centrifuged 10,000 $\mathrm{xg}$ for $10 \mathrm{~min}$. An aliquot ( $500 \mu \mathrm{L})$ was taken with $500 \mu \mathrm{L}$ of dthiocarbamate (DTC) in glass tubes. Reactants were left for $1 / 2 \mathrm{~h}$ at $37^{\circ} \mathrm{C}$. Test tubes containing reactant material was transferred to the ice-bath to terminate the reaction. After that, 2 $\mathrm{mL}$ of diluted $\mathrm{H}_{2} \mathrm{SO}_{4}$ was mixed slowly and left over for $1 / 2 \mathrm{~h}$ at $37^{\circ} \mathrm{C}$ in incubator. Extracted material was centrifuged at $12,000 \mathrm{xg}$. The shift in absorption was measured at $520 \mathrm{~nm}$ with the help of a spectrophotometer (Hitachi U-2910, Tokyo, Japan).

\section{Organic osmolytes}

\section{Total amino acids}

Free amino acid was quantified followed by Hamilton and Van-Slyke (1943) method. The $0.1 \mathrm{~g}$ of the leaf sample was grinded and immersed in a potassium phosphate buffer (SPB) overnight. After incubation, $1 \mathrm{~mL}$ of plant extract was transferred to $25 \mathrm{~mL}$ test tubes after adding $1 \mathrm{~mL}$ each of $10 \%$ ninhydrin and $2 \%$ of pyridine solution. The test tubes containing reactants were placed in boiling water bath for $1 \mathrm{~h}$. The final volume of samples was made to $25 \mathrm{~mL}$ by using deionized $\mathrm{H}_{2} \mathrm{O}$. Absorbance was recorded at $570 \mathrm{~nm}$ spectrophotometrically (Hitachi U-2910, Tokyo, Japan) and resulting absorbance were compared with the standard curve plotted for leucine.

\section{Soluble proteins}

Soluble proteins were appraised following Lowry et al (1951). Plant sample $(0.1 \mathrm{~g})$ was grounded in 50 $\mathrm{mM}$ sodium phosphate buffer (SPB) having $\mathrm{pH}$ 6.8. The extracted aliquot $(500 \mu \mathrm{L})$ was mixed in $0.3 \mathrm{~mL}$ of deionized $\mathrm{H}_{2} \mathrm{O}$ and $3 \mathrm{~mL}$ of Bio-Rad protein assay dye and vortexed for $15 \mathrm{~s}$. The absorbance was measured spectrophotometrically at $595 \mathrm{~nm}$ (Hitachi U-2910, Tokyo, Japan). Soluble proteins were estimated by comparing absorbance of samples with bovine serum albumin (BSA) using a standard value.

\section{Statistical analysis}

Minitab-19, software e (Minitab, LLC, State College, PA, USA) was used to analyze the data. The means values were compared by using the Tukey pairwise comparison test at $P \leq 0.05$ following an analysis of variance (ANOVA). The effect of $\mathrm{Ca}$ and $\mathrm{Cd}$ treatments was assessed by using a multivariate analysis (PCA by ggbiplot), correlation matrix (ggbiplot2) and heatmaps were plotted by customized code (pheatmap) by using R statistical software (R Core Team, 2019). Response curves under cadmium and calcium stress treatments were constructed by fitting a generalized linear model (GLM) in CONACO version 5 for windows. 


\section{Results}

\section{Plant growth traits}

Growth traits such as SL, RL, SFW, SDW, RFW, RDW and LA significantly decreased at Cd applied at150 $\mu \mathrm{m}$ concentration $(\mathrm{P} \leq 0.05)$. However, different levels of $\mathrm{Ca}$ significantly alleviated $\mathrm{Cd}$ toxicity and enhanced all growth traits. The increase in growth traits was more obvious in response to higher level of Ca applied at $10 \mathrm{mM}$ under $\mathrm{Cd}$ stress (Table 1). 
Table 1

Morphological characteristics of maize seedlings under $\mathrm{Ca}$ and $\mathrm{Cd}$ treatments

\begin{tabular}{|c|c|c|c|c|c|c|c|c|}
\hline \multicolumn{2}{|c|}{ Treatments } & \multirow{2}{*}{$\begin{array}{l}\text { SL } \\
(\mathrm{cm})\end{array}$} & \multirow{2}{*}{$\begin{array}{l}\mathrm{RL} \\
(\mathrm{cm})\end{array}$} & \multirow{2}{*}{$\begin{array}{l}\text { SFW (g } \\
\left.\text { plant }^{-1}\right)\end{array}$} & \multirow{2}{*}{$\begin{array}{l}\text { SDW (g } \\
\left.\text { plant }^{-1}\right)\end{array}$} & \multirow{2}{*}{$\begin{array}{l}\text { RFW (g } \\
\text { plant }^{-1} \text { ) }\end{array}$} & \multirow{2}{*}{$\begin{array}{l}\text { RDW (g } \\
\left.\text { plant }^{-1}\right)\end{array}$} & \multirow{2}{*}{$\begin{array}{l}\text { LA } \\
\left(\mathrm{Cm}^{2}\right)\end{array}$} \\
\hline Cd & $\mathrm{Ca}$ & & & & & & & \\
\hline \multirow[t]{6}{*}{$\begin{array}{l}C d-0 \\
\mu m\end{array}$} & $0 \mathrm{mM}$ & $\begin{array}{l}57.44 \\
\pm 5.71^{d}\end{array}$ & $\begin{array}{l}18.66 \\
\pm 1.32^{d}\end{array}$ & $\begin{array}{l}32.65 \pm \\
1.66^{d}\end{array}$ & $\begin{array}{l}3.12 \pm \\
0.13^{d}\end{array}$ & $\begin{array}{l}2.43 \pm \\
0.08^{d}\end{array}$ & $\begin{array}{l}0.26 \pm \\
0.02^{c}\end{array}$ & $\begin{array}{l}49.51 \pm \\
2.23^{d}\end{array}$ \\
\hline & $\begin{array}{l}0.5 \\
\mathrm{mM}\end{array}$ & $\begin{array}{l}66.22 \\
\pm 1.92^{c}\end{array}$ & $\begin{array}{l}23.61 \\
\pm 1.28^{d}\end{array}$ & $\begin{array}{l}40.72 \pm \\
1.82^{c}\end{array}$ & $\begin{array}{l}3.55 \pm \\
0.09^{d}\end{array}$ & $\begin{array}{l}3.09 \pm \\
0.16^{c}\end{array}$ & $\begin{array}{l}0.36 \pm \\
0.04^{c}\end{array}$ & $\begin{array}{l}57.79 \pm \\
0.86^{c}\end{array}$ \\
\hline & $1 \mathrm{mM}$ & $\begin{array}{l}66.22 \\
\pm 1.34^{\mathrm{C}}\end{array}$ & $\begin{array}{l}30.94 \\
\pm 0.80^{c}\end{array}$ & $\begin{array}{l}47.14 \pm \\
0.95^{c}\end{array}$ & $\begin{array}{l}4.46 \pm \\
0.15^{c}\end{array}$ & $\begin{array}{l}3.29 \pm \\
0.04^{\mathrm{c}}\end{array}$ & $\begin{array}{l}0.45 \pm \\
0.03^{b}\end{array}$ & $\begin{array}{l}60.99 \pm \\
4.06^{c}\end{array}$ \\
\hline & $\begin{array}{l}2.5 \\
\mathrm{mM}\end{array}$ & $\begin{array}{l}73.00 \\
\pm 0.84^{b}\end{array}$ & $\begin{array}{l}40.66 \\
\pm 2.47^{b}\end{array}$ & $\begin{array}{l}48.31 \pm \\
1.85^{c}\end{array}$ & $\begin{array}{l}4.54 \pm \\
0.36^{c}\end{array}$ & $\begin{array}{l}3.73 \pm \\
0.06^{b}\end{array}$ & $\begin{array}{l}0.56 \pm \\
0.02^{b}\end{array}$ & $\begin{array}{l}73.04 \pm \\
3.07^{b}\end{array}$ \\
\hline & $5 \mathrm{mM}$ & $\begin{array}{l}93.66 \\
\pm 2.95^{b}\end{array}$ & $\begin{array}{l}45.61 \\
\pm 3.11^{b}\end{array}$ & $\begin{array}{l}59.00 \pm \\
1.51^{b}\end{array}$ & $\begin{array}{l}5.87 \pm \\
0.22^{b}\end{array}$ & $\begin{array}{l}4.63 \pm \\
0.12^{\mathrm{a}}\end{array}$ & $\begin{array}{l}0.62 \pm \\
0.02^{a}\end{array}$ & $\begin{array}{l}75.91 \pm \\
3.60^{\mathrm{b}}\end{array}$ \\
\hline & $\begin{array}{l}10 \\
\mathrm{mM}\end{array}$ & $\begin{array}{l}105.7 \\
\pm 2.01^{\mathrm{a}}\end{array}$ & $\begin{array}{l}65.94 \\
\pm 2.84^{\mathrm{a}}\end{array}$ & $\begin{array}{l}81.91 \pm \\
3.19^{\mathrm{a}}\end{array}$ & $\begin{array}{l}7.75 \pm \\
0.10^{\mathrm{a}}\end{array}$ & $\begin{array}{l}5.60 \pm \\
0.11^{\mathrm{a}}\end{array}$ & $\begin{array}{l}0.66 \pm \\
0.03^{\mathrm{a}}\end{array}$ & $\begin{array}{l}95.87 \pm \\
2.34^{\mathrm{a}}\end{array}$ \\
\hline \multirow[t]{6}{*}{$\begin{array}{l}\text { Cd- } \\
150 \\
\mu \mathrm{m}\end{array}$} & $0 \mathrm{mM}$ & $\begin{array}{l}24.64 \\
\pm 2.38^{d}\end{array}$ & $\begin{array}{l}11.61 \\
\pm 2.22^{d}\end{array}$ & $\begin{array}{l}22.17 \pm \\
1.74^{\mathrm{d}}\end{array}$ & $\begin{array}{l}1.40 \pm \\
0.22^{\mathrm{c}}\end{array}$ & $\begin{array}{l}1.35 \pm \\
0.17^{c}\end{array}$ & $\begin{array}{l}0.14 \pm \\
0.02^{c}\end{array}$ & $\begin{array}{l}31.96 \pm \\
1.06^{\mathrm{d}}\end{array}$ \\
\hline & $\begin{array}{l}0.5 \\
\mathrm{mM}\end{array}$ & $\begin{array}{l}52.44 \\
\pm 3.75^{c}\end{array}$ & $\begin{array}{l}18.95 \\
\pm 0.80^{c}\end{array}$ & $\begin{array}{l}28.45 \pm \\
1.63^{d}\end{array}$ & $\begin{array}{l}1.61 \pm \\
0.03^{c}\end{array}$ & $\begin{array}{l}1.99 \pm \\
0.11^{c}\end{array}$ & $\begin{array}{l}0.16 \pm \\
0.01^{c}\end{array}$ & $\begin{array}{l}39.30 \pm \\
0.94^{d}\end{array}$ \\
\hline & $1 \mathrm{mM}$ & $\begin{array}{l}63.22 \\
\pm 3.74^{b}\end{array}$ & $\begin{array}{l}20.34 \\
\pm 2.35^{c}\end{array}$ & $\begin{array}{l}56.50 \pm \\
2.39^{c}\end{array}$ & $\begin{array}{l}1.91 \pm \\
0.39^{c}\end{array}$ & $\begin{array}{l}2.11 \pm \\
0.15^{b}\end{array}$ & $\begin{array}{l}0.21 \pm \\
0.01^{\mathrm{b}}\end{array}$ & $\begin{array}{l}53.40 \pm \\
5.49^{c}\end{array}$ \\
\hline & $\begin{array}{l}2.5 \\
\mathrm{mM}\end{array}$ & $\begin{array}{l}72.44 \\
\pm 2.92^{b}\end{array}$ & $\begin{array}{l}30.60 \\
\pm 1.45^{\mathrm{b}}\end{array}$ & $\begin{array}{l}73.11 \pm \\
2.11^{\mathrm{b}}\end{array}$ & $\begin{array}{l}2.81 \pm \\
0.28^{b}\end{array}$ & $\begin{array}{l}2.94 \pm \\
0.26^{\mathrm{a}}\end{array}$ & $\begin{array}{l}0.23 \pm \\
0.01^{\mathrm{b}}\end{array}$ & $\begin{array}{l}54.09 \pm \\
5.11^{\mathrm{c}}\end{array}$ \\
\hline & $5 \mathrm{mM}$ & $\begin{array}{l}82.77 \\
\pm 4.29^{a}\end{array}$ & $\begin{array}{l}35.10 \\
\pm 2.69^{b}\end{array}$ & $\begin{array}{l}77.96 \pm \\
5.19^{b}\end{array}$ & $\begin{array}{l}3.26 \pm \\
0.11^{b}\end{array}$ & $\begin{array}{l}3.10 \pm \\
0.18^{\mathrm{a}}\end{array}$ & $\begin{array}{l}0.32 \pm \\
0.03^{\mathrm{a}}\end{array}$ & $\begin{array}{l}62.96 \pm \\
1.46^{\mathrm{b}}\end{array}$ \\
\hline & $10 \mathrm{mM}$ & $\begin{array}{l}91.55 \\
\pm 3.83^{a}\end{array}$ & $\begin{array}{l}51.14 \\
\pm 3.16^{a}\end{array}$ & $\begin{array}{l}90.64 \pm \\
1.52^{\mathrm{a}}\end{array}$ & $\begin{array}{l}4.97 \pm \\
0.20^{a}\end{array}$ & $\begin{array}{l}3.11 \pm \\
0.09^{a}\end{array}$ & $\begin{array}{l}0.30 \pm \\
0.04^{\mathrm{a}}\end{array}$ & $\begin{array}{l}76.99 \pm \\
2.57^{a}\end{array}$ \\
\hline \multicolumn{9}{|c|}{$\begin{array}{l}\text { Means provided with error bars; in columns different letter indicates significance }(P \leq 0.05) \text { between } \\
\text { treatments }\end{array}$} \\
\hline \multicolumn{9}{|c|}{$\begin{array}{l}\text { Abbreviation: Shoot length (SL); Root length (RL); Shoot fresh weight (SFW); Shoot dry weight (SDW); } \\
\text { Root fresh weight (RFW); Root dry weight (RDW); Leaf area (LA) }\end{array}$} \\
\hline
\end{tabular}




\section{Photosynthetic Pigments}

Under $\mathrm{Cd}$ stress, a significant $(\mathrm{P} \leq 0.05)$ reduction occurred in the concentration of photosynthetic pigments of maize seedlings. Exogenously supplied Ca significantly increased photosynthetic pigments both in Cd stressed and non-stressed seedlings. Calcium applied at $10 \mathrm{mM}$ level was more beneficial in increasing chlorophyll and carotenoids contents of maize seedlings (Table 2). 
Table 2

Physiological traits of maize seedlings under various levels of $\mathrm{Ca}$ and $\mathrm{Cd}$ treatments

\begin{tabular}{|c|c|c|c|c|c|c|c|}
\hline \multicolumn{2}{|c|}{ Treatments } & \multirow{2}{*}{$\begin{array}{l}\text { Chl } a \\
\left(\mathrm{mg} \mathrm{g} \mathrm{g}^{-1}\right. \\
\text { FW) }\end{array}$} & \multirow{2}{*}{$\begin{array}{l}\text { Chl } b \\
\left(\mathrm{mg} \mathrm{g} \mathrm{g}^{-1}\right. \\
\mathrm{FW})\end{array}$} & \multirow{2}{*}{$\begin{array}{l}\text { Caro. } \\
\left(\mathrm{mg} \mathrm{g} \mathrm{g}^{-1}\right. \\
\text { FW) }\end{array}$} & \multirow{2}{*}{$\begin{array}{l}\text { T. Chl } \\
\left(\mathrm{mg} \mathrm{g} \mathrm{g}^{-1}\right. \\
\text { FW) }\end{array}$} & \multirow{2}{*}{$\begin{array}{l}\text { APX } \\
\text { (Units mg-1 } \\
\text { Pro) }\end{array}$} & \multirow{2}{*}{$\begin{array}{l}\text { POD } \\
\text { (Units } \mathrm{mg}^{-1} \\
\text { Pro) }\end{array}$} \\
\hline Cd & $\mathrm{Ca}$ & & & & & & \\
\hline \multirow[t]{6}{*}{$\begin{array}{l}\mathrm{Cd}-0 \\
\mu \mathrm{m}\end{array}$} & $0 \mathrm{mM}$ & $\begin{array}{l}9.76 \pm \\
0.25^{c}\end{array}$ & $\begin{array}{l}2.34 \pm \\
0.09^{c}\end{array}$ & $\begin{array}{l}0.35 \pm \\
0.01^{d}\end{array}$ & $\begin{array}{l}10.69 \pm \\
0.26^{c}\end{array}$ & $0.92 \pm 0.05^{c}$ & $0.55 \pm 0.11^{c}$ \\
\hline & $0.5 \mathrm{mM}$ & $\begin{array}{l}12.98 \pm \\
0.54^{c}\end{array}$ & $\begin{array}{l}2.84 \pm \\
0.23^{c}\end{array}$ & $\begin{array}{l}0.43 \pm \\
0.01^{c}\end{array}$ & $\begin{array}{l}11.43 \pm \\
0.75^{c}\end{array}$ & $1.02 \pm 0.04^{c}$ & $0.60 \pm 0.12^{c}$ \\
\hline & $1 \mathrm{mM}$ & $\begin{array}{l}16.56 \pm \\
0.84^{b}\end{array}$ & $\begin{array}{l}4.44 \pm \\
0.15^{b}\end{array}$ & $\begin{array}{l}0.48 \pm \\
0.02^{c}\end{array}$ & $\begin{array}{l}14.02 \pm \\
0.64^{b}\end{array}$ & $1.98 \pm 0.02^{b}$ & $0.73 \pm 0.04^{b}$ \\
\hline & $2.5 \mathrm{mM}$ & $\begin{array}{l}18.32 \pm \\
0.62^{\mathrm{a}}\end{array}$ & $\begin{array}{l}4.57 \pm \\
0.19^{b}\end{array}$ & $\begin{array}{l}0.51 \pm \\
0.02^{b}\end{array}$ & $\begin{array}{l}14.93 \pm \\
0.83^{b}\end{array}$ & $1.93 \pm 0.02^{b}$ & $0.78 \pm 0.03^{b}$ \\
\hline & $5 \mathrm{mM}$ & $\begin{array}{l}18.60 \pm \\
0.38^{a}\end{array}$ & $\begin{array}{l}5.48 \pm \\
0.18^{\mathrm{a}}\end{array}$ & $\begin{array}{l}0.52 \pm \\
0.03^{b}\end{array}$ & $\begin{array}{l}16.00 \pm \\
1.06^{\mathrm{a}}\end{array}$ & $1.98 \pm 0.06^{b}$ & $0.87 \pm 0.07^{a}$ \\
\hline & $10 \mathrm{mM}$ & $\begin{array}{l}19.26 \pm \\
0.76^{a}\end{array}$ & $\begin{array}{l}6.49 \pm \\
0.10^{\mathrm{a}}\end{array}$ & $\begin{array}{l}0.70 \pm \\
0.02^{\mathrm{a}}\end{array}$ & $\begin{array}{l}16.02 \pm \\
0.25^{a}\end{array}$ & $2.17 \pm 0.03^{a}$ & $0.91 \pm 0.06^{a}$ \\
\hline \multirow[t]{6}{*}{$\begin{array}{l}\text { Cd-150 } \\
\mu \mathrm{m}\end{array}$} & $0 \mathrm{mM}$ & $\begin{array}{l}3.63 \pm \\
0.22^{d}\end{array}$ & $\begin{array}{l}1.41 \pm \\
0.03^{\mathrm{c}}\end{array}$ & $\begin{array}{l}0.13 \pm \\
0.01^{c}\end{array}$ & $\begin{array}{l}5.02 \pm \\
0.23^{d}\end{array}$ & $1.27 \pm 0.03^{c}$ & $0.45 \pm 0.09^{d}$ \\
\hline & $0.5 \mathrm{mM}$ & $\begin{array}{l}5.33 \pm \\
0.19^{c}\end{array}$ & $\begin{array}{l}2.85 \pm \\
0.12^{c}\end{array}$ & $\begin{array}{l}0.17 \pm \\
0.01^{c}\end{array}$ & $\begin{array}{l}6.35 \pm \\
0.46^{c}\end{array}$ & $1.39 \pm 0.19^{b}$ & $0.72 \pm 0.02^{c}$ \\
\hline & $1 \mathrm{mM}$ & $\begin{array}{l}6.50 \pm \\
0.82^{c}\end{array}$ & $\begin{array}{l}3.08 \pm \\
0.08^{b}\end{array}$ & $\begin{array}{l}0.22 \pm \\
0.01^{b}\end{array}$ & $\begin{array}{l}7.25 \pm \\
0.45^{c}\end{array}$ & $2.01 \pm 0.04^{b}$ & $0.85 \pm 0.06^{b}$ \\
\hline & $2.5 \mathrm{mM}$ & $\begin{array}{l}10.72 \pm \\
0.47^{b}\end{array}$ & $\begin{array}{l}3.47 \pm \\
0.14^{b}\end{array}$ & $\begin{array}{l}0.27 \pm \\
0.01^{b}\end{array}$ & $\begin{array}{l}8.22 \pm \\
0.22^{b}\end{array}$ & $2.17 \pm 0.03^{a}$ & $0.82 \pm 0.06^{b}$ \\
\hline & $5 \mathrm{mM}$ & $\begin{array}{l}12.42 \pm \\
1.11^{\mathrm{b}}\end{array}$ & $\begin{array}{l}4.13 \pm \\
0.13^{\mathrm{a}}\end{array}$ & $\begin{array}{l}0.32 \pm \\
0.03^{\mathrm{a}}\end{array}$ & $\begin{array}{l}9.18 \pm \\
0.37^{b}\end{array}$ & $2.10 \pm 0.02^{a}$ & $1.10 \pm 0.01^{a}$ \\
\hline & $10 \mathrm{mM}$ & $\begin{array}{l}14.02 \pm \\
0.53^{\mathrm{a}}\end{array}$ & $\begin{array}{l}5.16 \pm \\
0.20^{\mathrm{a}}\end{array}$ & $\begin{array}{l}0.41 \pm \\
0.03^{\mathrm{a}}\end{array}$ & $\begin{array}{l}12.09 \pm \\
0.52^{\mathrm{a}}\end{array}$ & $2.22 \pm 0.01^{\mathrm{a}}$ & $1.12 \pm 0.06^{a}$ \\
\hline \multicolumn{8}{|c|}{$\begin{array}{l}\text { Means provided with error bars; in columns different letter indicates significance }(P \leq 0.05) \text { between } \\
\text { treatments }\end{array}$} \\
\hline $\begin{array}{l}\text { Abbrevic } \\
\text { Chl); Asc }\end{array}$ & $\begin{array}{l}\text { ns: Chlo } \\
\text { sate per }\end{array}$ & $\begin{array}{l}\text { Dhyll a }(C \\
\text { idase }(A \mid\end{array}$ & $\begin{array}{l}\text {; Chlor } \\
\text { Peroxid }\end{array}$ & $\begin{array}{l}\| l(\mathrm{Chl} l \\
(\mathrm{POD}) ; \mathrm{Pr}\end{array}$ & $\begin{array}{l}\text { arotenoi } \\
\text { (Pro) }\end{array}$ & ro); Total ch & phyll (T. \\
\hline
\end{tabular}


Mean values for antioxidant activity was higher in Cd stressed $(150 \mu \mathrm{M})$ as compared to non-stressed plants $(0 \mu \mathrm{M})$. However, the activity of APX significantly enhanced as levels of Ca increased both in nonstressed $(0 \mu \mathrm{M})$ and stressed plants $(150 \mu \mathrm{M})$. This increase was the maximum in response to Ca applied at $10 \mathrm{mM}$ concentration. Peroxidase activity showed the same trend as noted for APX under $\mathrm{Ca}$ and $\mathrm{Cd}$ treatments (Table 2).

\section{Anthocyanin and relative membrane permeability (RMP)}

Under Cd stress, maximum RMP values were observed indicating a high level of electrolyte leakage due to membrane damage. A significant $(P \leq 0.05)$ reduction was observed as the level of Ca increased (Fig 1). Anthocyanin contents under both treatments of $\mathrm{Cd}$ significantly increased as levels of $\mathrm{Ca}$ increased. Maximum anthocyanin contents were noticed at $10 \mathrm{mM}$ Ca concentration (Fig. 1). Cadmium applied at $150 \mu \mathrm{m}$ level and without any Ca supplementation had the most toxic effects as the highest electrolyte leakage was observed at this treatment level.

\section{Lipid peroxidation (LPX) and ROS}

The accumulation of $\mathrm{H}_{2} \mathrm{O}_{2}$ and MDA significantly increased in maize seedlings under $\mathrm{Cd}$ stress. However, the elevated levels of $\mathrm{Ca}$ significantly reduced the generation of $\mathrm{H}_{2} \mathrm{O}_{2}$ and LPX. The LPX in terms of MDA contents significantly decreased as the level of $\mathrm{Ca}$ increased in growth medium of the seedlings. The maximum decrease was observed under $10 \mathrm{mM}$ concentration of $\mathrm{Ca}$ (Fig. 1).

\section{Osmolytes}

Osmolyte (proteins and amino acids) production was significantly increased in maize seedling in stressed and non-stressed maize seedlings. Soluble proteins were significantly higher in non-stressed maize seedlings as the level of Ca increased (Fig 2). In Cd stressed seedlings (150 $\mu \mathrm{M})$, the concentration of soluble proteins significantly increased and the maximum was observed under $10 \mathrm{mM} \mathrm{Ca}$ concentration (Fig 2). Applications of Ca substantially increased the concentration of amino acids in both stressed and non-stressed seedlings and almost parallel results were observed as noted for soluble proteins (Fig 2).

\section{Ascorbic acid contents}

Ascorbic acid contents were substantially improved as Ca levels increased in maize seedlings under normal and stress conditions. Maximum values of ascorbic contents were observed under $10 \mathrm{mM}$ concentration of $\mathrm{Ca}$ in both stressed and non-stressed condition (Fig 2).

\section{Multivariate analysis}

\section{Principal component analysis (PCAs)}


PCAs results demonstrated high variations on the effects of $\mathrm{Cd}$ and $\mathrm{Ca}$ treatments among different growth and physio-biochemical traits of maize seedlings (Fig. 3). The first and second PCAs explained $75.8 \%$ and $17.2 \%$ (total $93 \%$ ) variation among treatments and seedlings characteristics. The major contributors to $150 \mu \mathrm{M}$ Cd level were amino acids (A-Ac), peroxidase (POD), $\mathrm{H}_{2} \mathrm{O}_{2}$, and RMP with high positive eigenvalues. The activity of antioxidative enzymes (POD, APX, ASC), photosynthetic pigments (Chl a), and growth traits significantly increased under Cd stress $(150 \mu \mathrm{M})$ with a higher concentration of $\mathrm{Ca}$ (C5-C6). Under lower levels of Ca i.e. C1 and C2, the ROS and RMP increased under Cd stress. The major principal components to control plants $(0 \mu \mathrm{M} \mathrm{Cd})$ were RFW, RDW, anthocyanin contents, $\mathrm{Chl} b, \mathrm{~T}$. Chl, carotenoids, and LPX with negative eigenvalues (Fig. 3). Cd stress significantly increased level of reactive oxygen species, while supplemented Ca significantly increased the antioxidative enzymes activity and growth parameters (Fig. 3).

\section{Correlation matrix}

In control plants, anthocyanin contents (Antho-C) was positively correlated with RFW, RDW, SL, LA, Caro, $\mathrm{Chl} b$ and TSP. The RMP, H2O2, and RMP were negatively correlated with RFW, RDW, Chl a, b, RL, LA, A-AC and APX (Fig. 4a). Under Cd stress, a highly positive correlation was assessed between POD, SFW, and ASC.A, APX, Chl a, SL, and RL. However, a strong negative correlation was assessed between $\mathrm{H}_{2} \mathrm{O}_{2}, \mathrm{RMP}$, and antioxidant enzymes under Cd-150 $\mu \mathrm{M}$ stress (Fig. 4b).

\section{Clustered heatmap}

A clustered heatmap was constructed to evaluate the effect of $\mathrm{Cd}$ and $\mathrm{Ca}$ treatments on the different traits as shown in Fig. 5. Under higher concentration of $\mathrm{Ca}(10 \mathrm{mM}), \mathrm{RMP}, \mathrm{H}_{2} \mathrm{O}_{2}$ and MDA showed significant reduction in response to 0 and $150 \mu \mathrm{M}$ concentration of $\mathrm{Cd}$ indicating a parallel response in both treatments. A noteworthy influence of $10 \mathrm{mM}$ level of Ca in non-stressed seedlings (0 $\mu \mathrm{M} C d$ ) was recorded with a greater increase in growth traits (RFW, RDW, SFW, SDW, SL, RL, LA), chlorophyll (Chl $a \& b, \mathrm{~T}$. Chl), organic osmolytes (TSP), anthocyanin contents (Antho.C) and ascorbic acid (ASC.A). All these traits were tightly grouped together and indicated high performance of $10 \mathrm{mM}$ level of Ca under non-stressed conditions. In Cd stressed (150 $\mathrm{MM})$ seedlings, $10 \mathrm{mM}$ level of Ca contributed to a significant increase in amino acids (A.AC), peroxidase (POD), ascorbic acid (ASC.A) and shoot fresh weight (SFW). Shoot length (SL), root length (RL), leaf area (LA), activity of ascorbate peroxidase (APX) and chlorophyll showed a strong and clear similarity and strongly clustered together. Antioxidants (APX and POD), ascorbic acid (ASC.A), anthocyanin contents (Antho.C) reduce the RMP, $\mathrm{H}_{2} \mathrm{O}_{2}$ and MDA and clustered together in same group. At highest level of $\mathrm{Ca}(10 \mathrm{mM})$, clustering and similarity indicated a high performance and a possible relationship between different traits under stress treatments.

\section{Response of different traits under stressed and non-stressed conditions}

In non-stressed conditions $(0 \mu \mathrm{M})$, a conspicuous positive response was observed for the growth traits (RL, SL, SFW, SDW, and LA) and chlorophyll (Chl $a$, Chl $b$ and T. Chl) as Ca levels increased (Fig 6a). 
Organic osmolytes (TAA, TSP), anthocyanin contents (AC) and ascorbic acid (A,ASc) showed a sharp positive response with increasing $\mathrm{Ca}$ regimes (Fig $6 \mathrm{~b}$ ). $\mathrm{H}_{2} \mathrm{O}_{2}, \mathrm{MDA}$ and RMP exhibits a strong negative response with an increase in Ca levels, however APX and POD exhibits increasing pattern in curve with

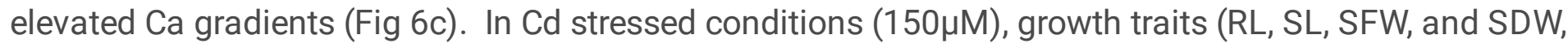
$\mathrm{LA}$ ) and chlorophyll (Chl $a, \mathrm{Chl} b$ and T.Chl) displayed a strong positive response and in response to $\mathrm{Ca}$ levels (Fig 6d). Concentration of TAA, TSP, AC and AA were the maximum with positive response (Fig 6e). A strong positive response was noted in activity of APX and POD along increasing Ca levels. In contrast, a strong negative response was assessed for $\mathrm{H}_{2} \mathrm{O}_{2}$, MDA and RMP with increase in Ca regimes (Fig 6f).

\section{Discussion}

Calcium plays an essential role in mitigation of abiotic stresses and protection from drastic impacts (Liang et al. 2005; Ahmad et al. 2015). It interacts with proteins like calmodulin to up-regulate gene expression and regulate movement of metal ions across membranes (Niu et al. 2017). The present work demonstrated that $\mathrm{Ca}$ significantly alleviated the toxic effect of $\mathrm{Cd}$ in maize by improving all growth traits. Furthermore, the alleviation of $\mathrm{Cd}$ toxicity was more obvious at higher treatment levels of $\mathrm{Ca}$ in stressed and non-stress plants. Previous studies revealed that $\mathrm{Ca}$ applications regulate the uptake of heavy metal ions as it competes for transporter sites on plasma membrane (Ahmad et al. 2015). Supplemented $\mathrm{Ca}^{2+}$ reduced $\mathrm{Cd}$ toxicity by enhancing growth traits as reported in other crops like in mustered (Ahmad et al. 2015) and rice (Farzadfar et al. 2013). Additionally, Ca reduced the toxic effect of nickel in rice seedlings (Aziz et al. 2015).

Reduction in growth traits under $\mathrm{Cd}$ toxicity is directly linked to the reduction of photosynthetic contents. As anticipated, the photosynthetic pigments significantly declined under $150 \mu \mathrm{M} C d$ treatment level. However, higher levels of Ca significantly improved carotenoids, total Chlorophyll (Chl), Chl $a, b$ pigments in maize seedlings under Cd stress (Table 2). Previously, the interactive effect between $\mathrm{Ca}$ and heavy metal was reported in some studies where exogenously applied Ca significantly prevented the damaging effects of Cd on photosynthetic pigments (Wu and Hendershot 2010; Aziz et al. 2015). Calcium is a divalent cation and shares many parallel physical properties (like $\mathrm{pH}$ ) with divalent heavy metals like $\mathrm{Cd}$, $\mathrm{Ni}$, and $\mathrm{Co}$ (Tian et al. 2011). Therefore, exogenously applied $\mathrm{Ca}$ ions through rooting medium can successfully restrict the uptake of $\mathrm{Cd}$ metal ions through competition for uptake and transport in plants (Tian et al. 2011). In current work, the enhanced amount of photosynthetic pigments in Cd treated maize seedlings seemed to be a direct effect of enhanced activities of anti-oxidative enzymes, and other protective molecules that reduced membrane damage (Zouari et al. 2016)

The improvement in the antioxidant defense system enables plants to alleviate the heavy metals toxicity (Aziz et al. 2015). In current study, APX and POD activities significantly improved in Ca treated plants under $\mathrm{Cd}$ toxicity. These results suggest that applied Ca can effectively alleviate $\mathrm{Cd}$ induced oxidative stress (Rodriguez-Serrano et al. 2009). Under heavy metal stress, Ca activates diverse protein kinases and strengthens antioxidant defense system (Siddiqui et al. 2012; Sudha and Ravishankar 2003). Tolerant plants had evolved an efficient antioxidant system to balance the concentration of reactive oxygen 
species (Mittler 2002; Huang et al. 2017). Enzymes like APX and POD also take part in the detoxification of free radicles and lead to sequestering of $\mathrm{H}_{2} \mathrm{O}_{2}$ (Sharma and Dietz 2006). APX is mainly localized in chloroplast, apoplast, cytosol, mitochondria, and peroxisome and POD in cell walls, cytosol, and vacuoles, Both APX and POD are mainly implicated to the scavenging of $\mathrm{H}_{2} \mathrm{O}_{2}$ (Mittler 2002). Their efficiency enhanced during $\mathrm{Cd}$ stresses and that greatly imparts stress tolerance and modulates the physiological process in maize seedlings in this study (Ahmad et al. 2010; Siddiqui et al. 2011).

Plants exposed to metal stress showed alterations in cell membrane permeability (RMP) and consequently cell loses membranes integrity (O'Lexy et al. 2018). Cell membrane integrity is considered as a tool to regulate the ionic movements and use as a selection criteria to quantify damage magnitude. In current results, the relative RMP markedly increased under Cd stress. However, the RMP significantly was markedly reduced by the $C$ a treatments that alleviated the damaging consequences of $\mathrm{Cd}$. In plants exposed to Cd stress, relative membrane permeability (RMP) substantially increased and caused membrane impairments (Mishra et al. 2019). Under Cd stress, supplemented Ca decrease relative membrane permeability (electrolyte leakage) showing the role of Ca played in membrane stability (Javed et al. 2017). Ca mainly stabilizes the membrane integrity and also controls the movement of divalent cations and prevent solute leakage by reducing peroxidation of lipids (Antosiewicz and Hennig 2004; Farzadfar et al. 2013).

The excessive accumulation of both MDA and $\mathrm{H}_{2} \mathrm{O}_{2}$ in under metal stresses damages biomolecules by excessive lipid peroxidation, degrades membranes, decreases photosynthesis and hampered the activity of other essential enzymes (Mittler 2002). Plants enhance antioxidant system to deplete the ROS which ultimately reduce oxidative stress generated by high metal concentrations (Karuppanapandian and Manoharan 2008). The Ca applications as observed in this study, improved activities of various antioxidants (enzymatic or non-enzymatic) and reduced the level of $\mathrm{H}_{2} \mathrm{O}_{2}$ and lipid peroxidation (Candan and Tarhan 2005; Siddiqui et al. 2012). Calcium also up-regulates genes which are responsible to encode the antioxidant under oxidative stress (Jiang and Huang 2001). In present work, the level of ROS increased under $\mathrm{Cd}$ stress, however, the addition of $\mathrm{Ca}$ considerably reduced the production of ROS in maize seedlings (Fig. 1).

The anthocyanin contents remarkably increased in present study that was more pronounced in highest levels of Ca (Fig. 1)that is are parallel to many previous findings (Sudha and Ravishankar 2003; Yamdech et al. 2012). High level of anthocyanin regulates heavy metal transport toward the vacuole and sequestration (Amiri et al. 2012). Exogenously applied calcium is reported to reduced Cd toxicity by stimulating the synthesis of glutathione-S-transferase (GST) enzyme to increase anthocyanin contents that in turn ameliorates the oxidative stress by scavenging the free radicals (Amiri et al. 2012).

Heavy metal stress causes determinal changes in cellular structures and cause osmotic stress (RucińskaSobkowiak 2016)Plants mitigate with osmotic stress by accumulating the lower or higher weight osmolytes that do not hinder the functioning of important metabolites (Rhodes et al. 2002). Osmolytes primarily reduces water potential and ensure the water balance (Wang et al. 2003), protects subcellular 
structures, and reduces oxidative damage (Slama et al. 2008). Amino acids act as organic osmolytes and participate in osmotic adjustments, stabilize proteins in membranes (Lee et al. 2008), ion homeostasis (Gleeson et al. 2005), scavenges the ROS and neutralize the redox potential during oxidative stress caused by noxious heavy metals (Lee et al. 2008). In the present studies, the seedlings showed more accumulation of osmolytes under application of Ca (Fig. 2). Ascorbic acids (ASc) are non-enzymatic antioxidant enzymes, acts as a cofactor for many important enzymes and accumulate in leaves (Ahmad et al. 2010). It serves a defensive role during oxidative stress and reduces the $\mathrm{H}_{2} \mathrm{O}_{2}$ and detoxifies the free radicals (Türkan et al. 2005). In present study, ascorbic acid in maize seedlings was significantly enhanced by the addition of $\mathrm{Ca}$ (Fig. 2).

\section{Conclusions}

In conclusion, $\mathrm{Cd}$ induced oxidative stress caused negative influences on growth and physio-biochemical traits of plants. The addition of Ca significantly enhanced the growth and physio-biochemical traits. Exogenously applied $\mathrm{Ca}$ ameliorated the oxidative stress by increasing the APX and POD activities, and ascorbic acid contents to withstand $\mathrm{Cd}$ toxicity and increased tolerance. Calcium treatments significantly $(P \leq 0.05)$ reduced the ROS by enhancing the antioxidant enzymes. The amounts of osmolytes (amino acids and proteins) improved significantly for osmotic adjustments. In conclusion, exogenous applications of $\mathrm{Ca}$ can mitigate the adverse effects caused by $\mathrm{Cd}$ and enabled maize seedlings to thrive as evident by increased osmolytes, antioxidants, and growth traits.

\section{Abbreviations}

Melanoaldehyde (MDA); Relative membrane permeability (RMP); Hydrogen per oxide $\left(\mathrm{H}_{2} \mathrm{O}_{2}\right)$; Root fresh weight (RFW); Root length (RL); Total chlorophyll (T. Chl); Shoot dry weight (SDW); Ascorbic acid (ASC.A); Carotenoids (Caro); Anthocyanin contents (Antho-C, ACC); Chlorophyll $b(\mathbf{C h l} b)$; Chlorophyll $a(C h l ~ a)$; Total soluble proteins (TSP); Leaf area (LA); Root dry weight (RDW); Peroxidase (POD); Shoot fresh weight (SFW); Ascorbate per oxidase (APX); Amino Acids (A-AC); Sodium phosphate buffer (SPB)

\section{Declarations}

Ethics approval and consent to participate: Not applicable.

Consent for publication: The authors confirm that the final version of the manuscript has been reviewed, approved, and agreed for publication by all authors.

Data availability: Authors confirm that all relevant data are included in the article and would be ready to share the raw data upon request.

Funding statement: Project is not funded by any organization.

\section{Author contribution}


Muhammad Kaleem: This manuscript was part of his MSc Thesis research work submitted to Government College University, Faisalabad

Iqbal Hussain: acted as major research supervisor of the first author

Mansoor Hameed, Muhammad Sajid Aqeel Ahmad: Research planning and conceptualization

Anam Mehmood, Muhammad Faisal Maqsood: Data interpretation and manuscript compilation

Ummar Iqbal, Syed Mohsan Raza Shah: Biochemical analysis and data collection

Waseem Ashfaq, Zunaira Anwar: Data analysis and visualization

Declaration of interest: No conflicts of interest

Acknowledgment: This manuscript was part of his MSc Thesis research work submitted to Government College University, Faisalabad (GCUF). The authors acknowledge the support provided by the GCUF for planning, execution and analysis of this research work.

\section{References}

1. Ahmad P, Jaleel CA, Salem MA, Nabi G, Sharma S (2010) Roles of enzymatic and nonenzymatic antioxidants in plants during abiotic stress Crit Rev Biotechnol 30:161-175. https://doi.org/10.3109/07388550903524243

2. Ahmad P, Sarwat M, Bhat NA, Wani MR, Kazi AG, Tran L-SP (2015) Alleviation of cadmium toxicity in Brassica juncea L.(Czern. \& Coss.) by calcium application involves various physiological and biochemical strategies PLoS One 10. https://doi.org/10.1371/journal.pone.0114571

3. Amiri J, Entesari S, Delavar K, Saadatmand M, Rafie NA (2012) The effect of silicon on cadmium stress in Echium amoenum World Acad Sci Eng Technol 62:242-245.

4. Antosiewicz DM, Hennig J (2004) Overexpression of LCT1 in tobacco enhances the protective action of calcium against cadmium toxicity Environ Pollut 129:237-

245. https://doi.org/10.1016/j.envpol.2003.10.025

5. Arnon DI (1949) Copper enzymes in isolated chloroplasts. Polyphenoloxidase in Beta vulgaris Plant Physiol 24: 1. https://doi.org/10.1104/pp.24.1.1

6. Asgher M, Khan NA, Khan MIR, Fatma M, Masood A (2014) Ethylene production is associated with alleviation of cadmium-induced oxidative stress by sulfur in mustard types differing in ethylene sensitivity. Ecotoxicol Environ Saf 106:54-61. https://doi.org/10.1016/j.ecoenv.2014.04.017

7. Aziz H, Sabir M, Ahmad HR, Aziz T, Zia-ur-Rehman M, Hakeem KR, Ozturk M (2015) Alleviating effect of calcium on nickel toxicity in rice. CLEAN-Soil Air Water 43:901909. https://doi.org/10.1002/clen.201400085 
8. Bellincampi D, Dipierro N, Salvi G, Cervone F, De Lorenzo G (2000) Extracellular $\mathrm{H}_{2} \mathrm{O}_{2}$ induced by oligogalacturonides is not involved in the inhibition of the auxin-regulated rolB gene expression in tobacco leaf explants Plant Physiol 122: 1379-1386. https://doi.org/10.1104/pp.122.4.1379

9. Boomsma CR, Vyn TJ (2008) Maize drought tolerance potential improvements through arbuscular mycorrhizal symbiosis? Field Crops Res 108:14-31. https://doi.org/10.1016/j.fcr.2008.03.002

10. Candan N, Tarhan L (2005) Effects of calcium, stress on contents of chlorophyll and carotenoid, LPO levels, and antioxidant enzyme activities in Mentha. J Plant Nutr 28:127-

139. https://doi.org/10.1081/PLN-200042192

11. Chen G-X, Asada K (1989) Ascorbate peroxidase in tea leaves occurrence of two isozymes and the differences in their enzymatic and molecular properties Plant Cell Physiol 30:987-998.

12. Davis BH. 1976. Carotenoids. In Goodwin TW, editor. Chemistry and biochemistry of plant pigments. 2nd ed. 518 London Academic Press Inc. p. 38-165.

13. Dourado M, Franco M, Peters L, Martins P, Souza L, Piotto F, Azevedo R (2015) Antioxidant enzymes activities of Burkholderia spp. Strains-oxidative responses to Ni toxicity Environ Sci Pollut Res 22:19922-19932. https://doi.org/10.1007/s11356-015-5204-1

14. El-Beltagi HS, Mohamed HI (2013) Alleviation of cadmium toxicity in Pisum sativum L. seedlings by calcium chloride Notulae Botanicae Horti Agrobotanici Cluj-Napoca 41:157-168.

15. Farzadfar S, Zarinkamar F, Modarres-Sanavy SAM, Hojati M (2013) Exogenously applied calcium alleviates cadmium toxicity in Matricaria chamomilla L. plants. Environ Sci Pollut Res 20: 14131422. https://doi.org/10.1007/s11356-012-1181-9

16. Gallego SM, Pena LB, Barcia RA, Azpilicueta CE, lannone MF, Rosales EP, Zawoznik MS, Groppa MD, Benavides MP (2012) Unravelling cadmium toxicity and tolerance in plants insight into regulatory mechanisms. Environ Exp Bot 83:33-46. https://doi.org/10.1016/j.envexpbot.2012.04.006

17. Giusti MM, Wrolstad RE (2001) Current protocols in food analytical chemistry. Current protocols in food analytical chemistry, I'. pp. F 1, F1

18. Gleeson D, Lelu-Walter M-A, Parkinson M (2005) Overproduction of proline in transgenic hybrid larch (Larix x leptoeuropaea (Dengler)) cultures renders them tolerant to cold, salt and frost. Molecul Breed 15:21-29. https://doi.org/10.1007/s11032-004-1363-3

19. Goix S, Lévêque T, Xiong T-T, Schreck E, Baeza-Squiban A, Geret F, Uzu G, Austruy A, Dumat C (2014) Environmental and health impacts of fine and ultrafine metallic particles assessment of threat scores. Environ Res 133:185-194. https://doi.org/10.1016/j.envres.2014.05.015

20. Goliber TE (1989) Gravitational stress and lignification in aerial vs. submerged shoots of Hippuris vulgaris. Physiol Plant 75:355-361. https://doi.org/10.1111/j.1399-3054.1989.tb04638.x

21. Gratão PL, Monteiro CC, Tezotto T, Carvalho RF, Alves LR, Peters LP, Azevedo RA (2015) Cadmium stress antioxidant responses and root-to-shoot communication in grafted tomato plants. Biometals 28:803-816. https://doi.org/10.1007/s10534-015-9867-3 
22. Grzybowska EA (2018) Calcium-binding proteins with disordered structure and their role in secretion, storage, and cellular signaling. Biomolecules 8:42. https://doi.org/10.3390/biom8020042

23. Hamilton P, Van Slyke D (1943) Amino acid determination with ninhydrin. J. biol. Chem 150, 231-250

24. Heath RL, Packer $L$ (1968) Photoperoxidation in isolated chloroplasts I. Kinetics and stoichiometry of fatty acid peroxidation. Arch Biochem Biophys 125:189-198. https://doi.org/10.1016/00039861(68)90654-1

25. Huang D, Gong X, Liu Y, Zeng G, Lai C, Bashir H, Zhou L, Wang D, Xu P, Cheng M (2017) Effects of calcium at toxic concentrations of cadmium in plants. Planta $245: 863-$

873. https://doi.org/10.1007/s00425-017-2664-1

26. Huybrechts M, Cuypers A, Deckers J, Iven V, Vandionant S, Jozefczak M, Hendrix S (2019) Cadmium and plant development An agony from seed to seed. Int J Mol Sci 20:3971. https://doi.org/10.3390/ijms20163971

27. Javed MT, Akram MS, Tanwir K, Chaudhary HJ, Ali Q, Stoltz E, Lindberg S (2017) Cadmium spiked soil modulates root organic acids exudation and ionic contents of two differentially Cd tolerant maize (Zea mays L.) cultivars. Ecotoxicol Environ Saf 141:216225. https://doi.org/10.1016/j.ecoenv.2017.03.027

28. Jiang $Y$, Huang B (2001) Effects of calcium on antioxidant activities and water relations associated with heat tolerance in two cool-season grasses. $J$ Exp Bot52: 341-

349. https://doi.org/10.1093/jexbot/52.355.341

29. Karuppanapandian T, Manoharan K (2008) Uptake and translocation of tri-and hexa-valent chromium and their effects on black gram (Vigna mungo L. Hepper cv. Co4) roots. J Plant Biol 51: 192-201. https://doi.org/10.1007/BF03030698

30. Kováčik J, Babula P, Hedbavny J, Švec P (2014) Manganese-induced oxidative stress in two ontogenetic stages of chamomile and amelioration by nitric oxide. Plant Sci 215:110. https://doi.org/10.1016/j.plantsci.2013.10.015

31. Lee G, Carrow RN, Duncan RR, Eiteman MA, Rieger MW (2008) Synthesis of organic osmolytes and salt tolerance mechanisms in Paspalum vaginatum. Environ Exp Bot 63:19-

27. https://doi.org/10.1016/j.envexpbot.2007.10.009

32. Liang Y, Wong J, Wei L (2005) Silicon-mediated enhancement of cadmium tolerance in maize (Zea mays L.) grown in cadmium contaminated soil. Chemosphere 58:475483. https://doi.org/10.1016/j.chemosphere.2004.09.034

33. Liu H, Hussain S, Peng S, Huang J, Cui K, Nie L (2014) Potentially toxic elements concentration in milled rice differ among various planting patterns. Field Crop Res 168:19-

26. https://doi.org/10.1016/j.fcr.2014.08.007

34. Lowry OH, Rosebrough NJ, Farr AL, Randall RJ (1951) Protein measurement with the Folin phenol reagent. J Biol Chem 193: 265-275.

35. Lwalaba JLW, Zvobgo G, Fu L, Zhang X, Mwamba TM, Muhammad N, Mundende RPM, Zhang G (2017) Alleviating effects of calcium on cobalt toxicity in two barley genotypes differing in cobalt 
tolerance. Ecotoxicol Environ Saf 139:488-495. https://doi.org/10.1016/j.ecoenv.2017.02.019

36. Maleki M, Ghorbanpour M, Kariman K (2017) Physiological and antioxidative responses of medicinal plants exposed to heavy metals stress. Plant Gene 11:247-

254. https://doi.org/10.1016/j.plgene.2017.04.006

37. Malik Z, Afzal S, Danish M, Abbasi GH, Bukhari SAH, Khan MI, Dawood M, Kamran M, Soliman MH, Rizwan M (2020) Role of Nitric Oxide and Calcium Signaling in Abiotic Stress Tolerance in Plants. Protective Chemical Agents in the Amelioration of Plant Abiotic Stress Biochemical and Molecular Perspectives, 563-581. https://doi.org/10.1002/9781119552154.ch28

38. Mishra B, Sangwan RS, Mishra S, Jadaun JS, Sabir F, Sangwan NS (2014) Effect of cadmium stress on inductive enzymatic and nonenzymatic responses of ROS and sugar metabolism in multiple shoot cultures of Ashwagandha (Withania somnifera Dunal). Protoplasma 251:10311045. https://doi.org/10.1007/s00709-014-0613-4

39. Mishra S, Bharagava RN, More N, Yadav A, Zainith S, Mani S, Chowdhary P (2019) Heavy metal contamination an alarming threat to environment and human health, Environmental biotechnology For sustainable future. Springer, pp. 103-125

40. Mittler R (2002) Oxidative stress, antioxidants and stress tolerance. Trend Plant Sci 7:405410. https://doi.org/10.1016/S1360-1385(02)02312-9

41. Nino H, Shah W (1986) Vitamins In Fundamentals of Clinical Chemistry. Tietz, NW. WB Saunders, Philadelphia

42. Niu L, Liao W (2016) Hydrogen peroxide signaling in plant development and abiotic responses crosstalk with nitric oxide and calcium. Front Plant Sci

7:230. https://doi.org/10.3389/fpls.2016.00230

43. Niu L, Yu J, Liao W, Yu J, Zhang M, Dawuda MM (2017) Calcium and calmodulin are involved in nitric oxide-induced adventitious rooting of cucumber under simulated osmotic stress. Front Plant Sci 8:1684. https://doi.org/10.3389/fpls.2017.01684

44. Nogueirol RC, Monteiro FA, Gratão PL, da Silva BKdA, Azevedo RA (2016) Cadmium application in tomato nutritional imbalance and oxidative stress. Water Air Soil Pollut 227:120. https://doi.org/10.1007/s11270-016-2895-y

45. Nuss ET, Tanumihardjo SA (2010) Maize a paramount staple crop in the context of global nutrition. Comprehensive reviews in food science and food safety 9: 417-436. https://doi.org/10.1111/j.15414337.2010.00117.x

46. O'Lexy R, Kasai K, Clark N, Fujiwara T, Sozzani R, Gallagher KL (2018) Exposure to heavy metal stress triggers changes in plasmodesmatal permeability via deposition and breakdown of callose. $\mathrm{J}$ Exp Bot 69:3715-3728. https://doi.org/10.1093/jxb/ery171

47. Rady MM, Elrys AS, El-Maati MFA, Desoky E-SM (2019) Interplaying roles of silicon and proline effectively improve salt and cadmium stress tolerance in Phaseolus vulgaris plant. Plant Physiol Biochem 139, 558-568. https://doi.org/10.1016/j.plaphy.2019.04.025 
48. Rani P, Chakraborty M, Sah RP (2015) Identification and genetic estimation of nutritional parameters of QPM hybrids suitable for animal feed purpose. Range Manage Agrofor 36:175-182.

49. Rhodes D, Nadolska-Orczyk A, Rich P (2002) Salinity, osmolytes and compatible solutes, Salinity Environment-plants-molecules. Springer, pp. 181-204. https://doi.org/10.1007/0-306-48155-3_9

50. Rodríguez-Serrano M, Romero-Puertas MC, Pazmino DM, Testillano PS, Risueño MC, Luis A, Sandalio LM (2009) Cellular response of pea plants to cadmium toxicity cross talk between reactive oxygen species, nitric oxide, and calcium. Plant Physiol 150:229-243.

51. Rucińska-Sobkowiak R (2016) Water relations in plants subjected to heavy metal stresses. Acta Physiol Plant 38: 1-13. https://doi.org/10.1007/s11738-016-2277-5

52. Sah R, Chakraborty M, Prasad K, Pandit M, Tudu V, Chakravarty M, Narayan S, Rana M, Moharana D (2020) Impact of water deficit stress in maize Phenology and yield components. Sci Rep 10:115. https://doi.org/10.1038/s41598-020-59689-7

53. Sharma SS, Dietz K-J (2006) The significance of amino acids and amino acid-derived molecules in plant responses and adaptation to heavy metal stress. J Exp Bot 57:711-

726. https://doi.org/10.1093/jxb/erj073

54. Siddiqui MH, Al-Whaibi MH, Basalah MO (2011) Interactive effect of calcium and gibberellin on nickel tolerance in relation to antioxidant systems in Triticum aestivum L. Protoplasma 248:503511. https://doi.org/10.1007/s00709-010-0197-6

55. Siddiqui MH, Al-Whaibi MH, Sakran AM, Basalah MO, Ali HM (2012) Effect of calcium and potassium on antioxidant system of Vicia faba L. under cadmium stress. Int J Mol Sci 13:66046619. https://doi.org/10.3390/ijms13066604

56. Slama I, Ghnaya T, Savouré A, Abdelly C (2008) Combined effects of long-term salinity and soil drying on growth, water relations, nutrient status and proline accumulation of Sesuvium portulacastrum. Comp Ren Biolog 331:442-451. https://doi.org/10.1016/j.crvi.2008.03.006

57. Song J, Chen M, Feng G, Jia Y, Wang B, Zhang F (2009) Effect of salinity on growth, ion accumulation and the roles of ions in osmotic adjustment of two populations of Suaeda salsa. Plant and Soil 314:133-141. https://doi.org/10.1007/s11104-008-9712-3

58. Srivastava RK, Pandey P, Rajpoot R, Rani A, Gautam A, Dubey R (2015) Exogenous application of calcium and silica alleviates cadmium toxicity by suppressing oxidative damage in rice seedlings. Protoplasma 252:959-975. https://doi.org/10.1007/s00709-014-0731-z

59. Sudha G, Ravishankar GA (2003) The role of calcium channels in anthocyanin production in callus cultures of Daucus carota. Plant Growth Reg 40:163-

169. https://doi.org/10.1023/A:1024298602617

60. Tian S, Lu L, Zhang J, Wang K, Brown P, He Z, Liang J, Yang X (2011) Calcium protects roots of Sedum alfredii $\mathrm{H}$. against cadmium-induced oxidative stress. Chemosphere 84:6369. https://doi.org/10.1016/j.chemosphere.2011.02.054

61. Türkan I, Bor M, Özdemir F, Koca H (2005) Differential responses of lipid peroxidation and antioxidants in the leaves of drought-tolerant P. acutifolius Gray and drought-sensitive $P$. vulgaris $\mathrm{L}$. 
subjected to polyethylene glycol mediated water stress. Plant Sci 168:223-

231. https://doi.org/10.1016/j.plantsci.2004.07.032

62. Vigani G, Costa A (2019) Harnessing the new emerging imaging technologies to uncover the role of $\mathrm{Ca}^{2+}$ signalling in plant nutrient homeostasis. Plant Cell Environ 42:28852901. https://doi.org/10.1111/pce.13611

63. Wang H-Y, Huang Y-C, Chen S-F, Yeh K-W (2003) Molecular cloning, characterization and gene expression of a water deficiency and chilling induced proteinase inhibitor I gene family from sweet potato (Ipomoea batatas Lam.) leaves. Plant Sci 165:191-203. https://doi.org/10.1016/S01689452(03)00158-4

64. Wu Y, Hendershot WH (2010) The effect of calcium and pH on nickel accumulation in and rhizotoxicity to pea (Pisum sativum L.) root-empirical relationships and modeling. Environ Pollut 15:1850-1856. https://doi.org/10.1016/j.envpol.2009.10.046

65. Yamdech R, Aramwit P, Kanokpanont S (2012) Stability of Anthocyanin in Mulberry Fruits Extract Adsorbed on Calcium Alginate Beads, International Conference Chulalongkorn University, Bangkok Thailand.

66. Yang G, Rhodes D, Joly RJ (1996) Effects of high temperature on membrane stability and chlorophyll fluorescence in glycinebetaine-deficient and glycinebetaine-containing maize lines. Funct Plant Biol 23:437-443. https://doi.org/10.1071/PP9960437

67. Zouari M, Ahmed CB, Elloumi N, Bellassoued K, Delmail D, Labrousse P, Abdallah FB, Rouina BB (2016) Impact of proline application on cadmium accumulation, mineral nutrition and enzymatic antioxidant defense system of Olea europaea L. cv Chemlali exposed to cadmium stress. Ecotoxicol Environ Saf 128, 195-205. https://doi.org/10.1016/j.ecoenv.2016.02.024

\section{Figures}



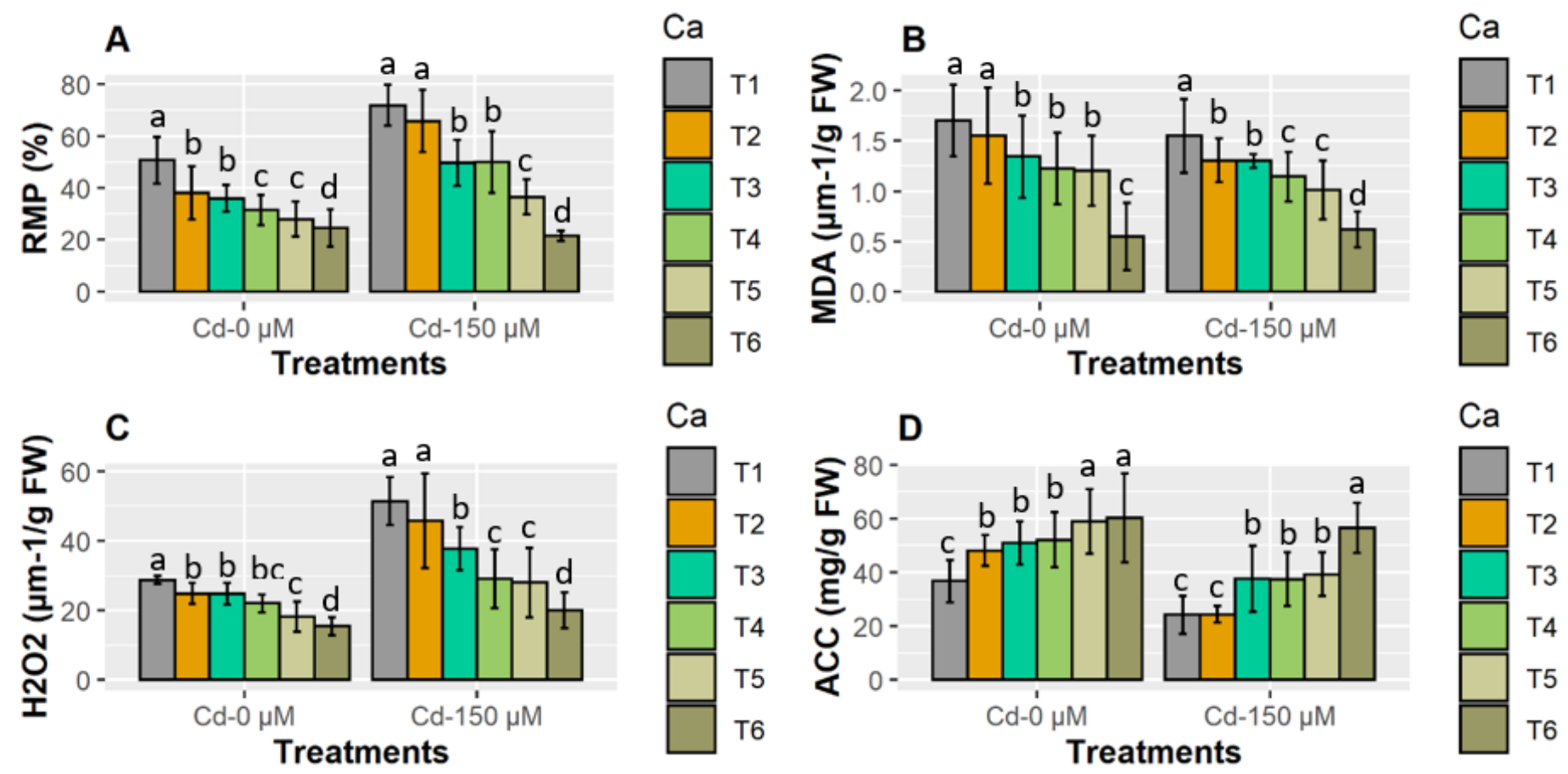

Figure 1

Effect of calcium (Ca; T1-0 mM, T2-0.5 mM, T3-1 mM, T4-2.5 mM, T5-5 mM, T6-10 mM) and cadmium (Cd) treatments on the A) relative membrane permeability (RMP), B) melanoaldehyde contents, C) H2O2, and $D$ ) anthocyanine contents (ACC) of maize seedlings. Means \pm SE provided with error bars; different letter indicates significance $(\mathrm{P} \leq 0.05)$ between $\mathrm{Ca}$ and $\mathrm{Cd}$ treatments.

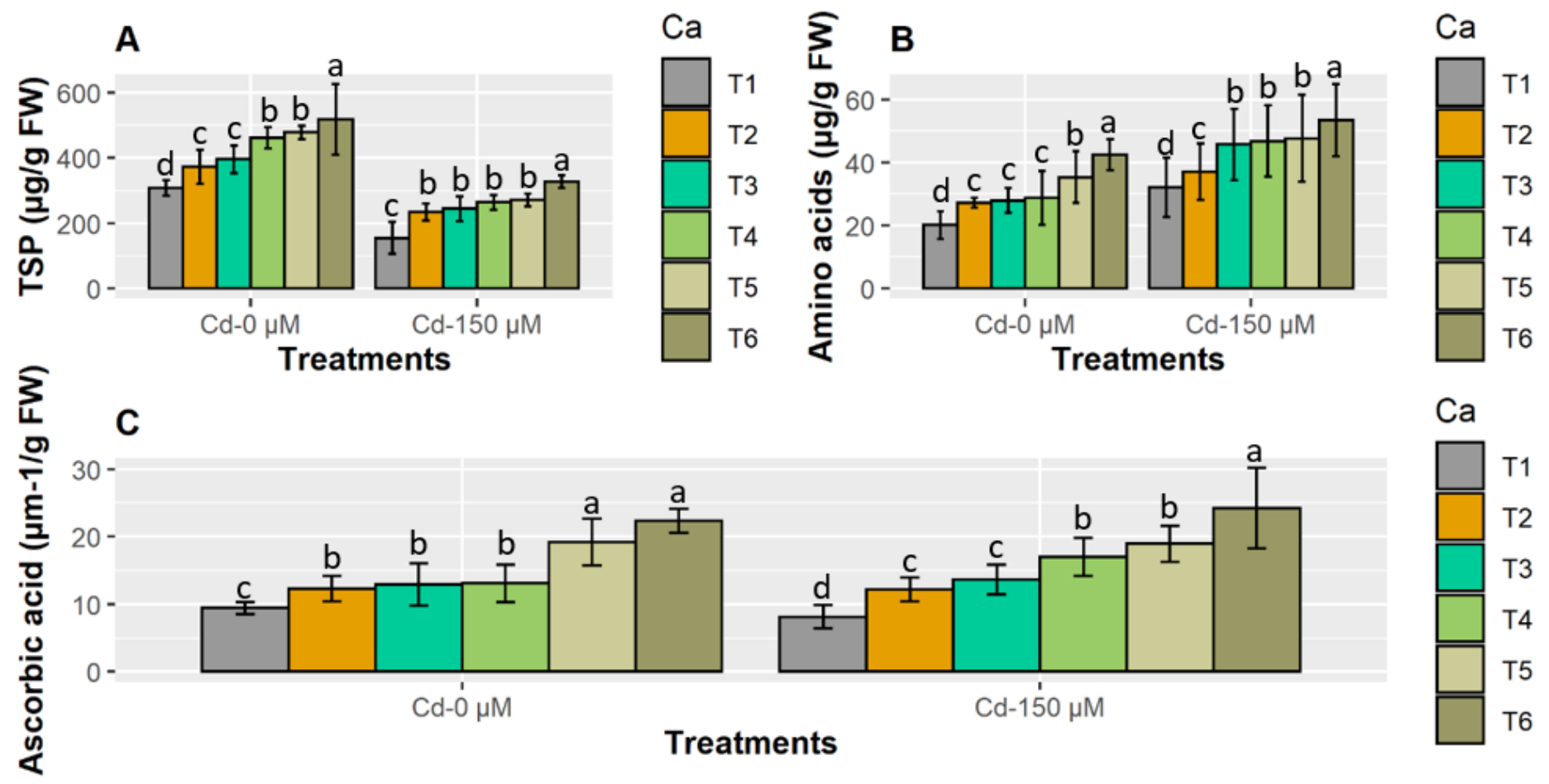

Figure 2 
Effect of calcium (Ca; T1-0 mM, T2-0.5 mM, T3-1 mM, T4-2.5 mM, T5-5 mM, T6-10 mM) and cadmium (Cd) treatments on the A) toatl soluble protiens, B) amino acids and C) ascorbic acid contents of maize seedlings. Means \pm SE provided with error bars; different letter indicates significance $(P \leq 0.05)$ between $\mathrm{Ca}$ and Cd treatments.

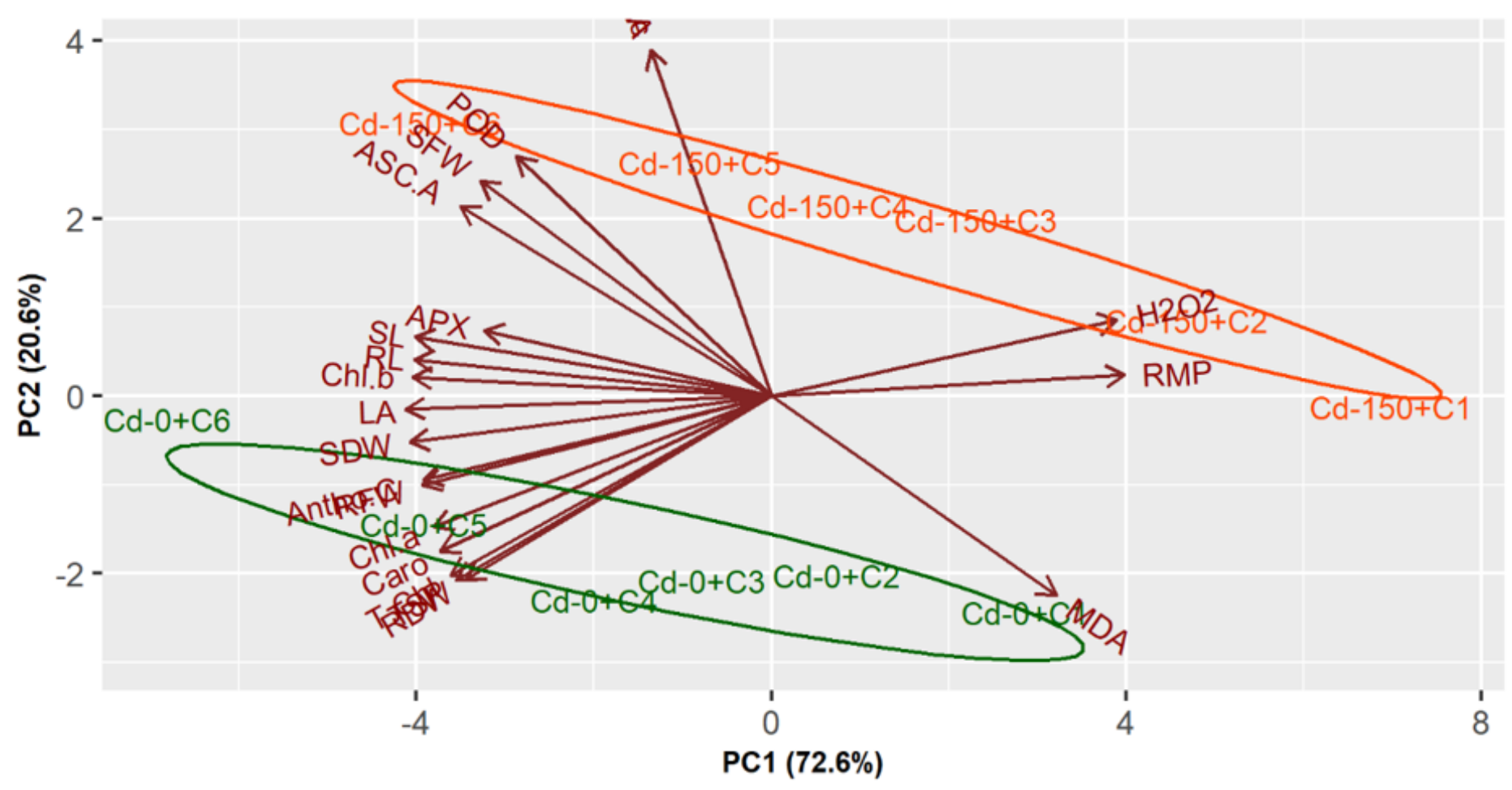

Origin $\rightarrow \mathrm{Cd}-0 \mu \mathrm{M}$ - $\mathrm{Cd}-150 \mu \mathrm{M}$

\section{Figure 3}

PCA biplot for growth and physio-biochemical traits under $\mathrm{Cd}$ and calcium treatments Abbreviations: Shoot length (SL); Root length (RL); Shoot fresh weight (SFW); Shoot dry weight (SDW); Root fresh weight (RFW); Root dry weight (RDW); Leaf area (LA); Chlorophyll a (Chl a); Chlorophyll b (Chl b); Carotenoids (Caro); Total chlorophyll (T. Chl); Ascorbate per oxidase (APX); Peroxidase (POD); relative membrane permeability (RMP); hydrogen per oxide (H2O2) and ascorbic acid (ASC.A). 


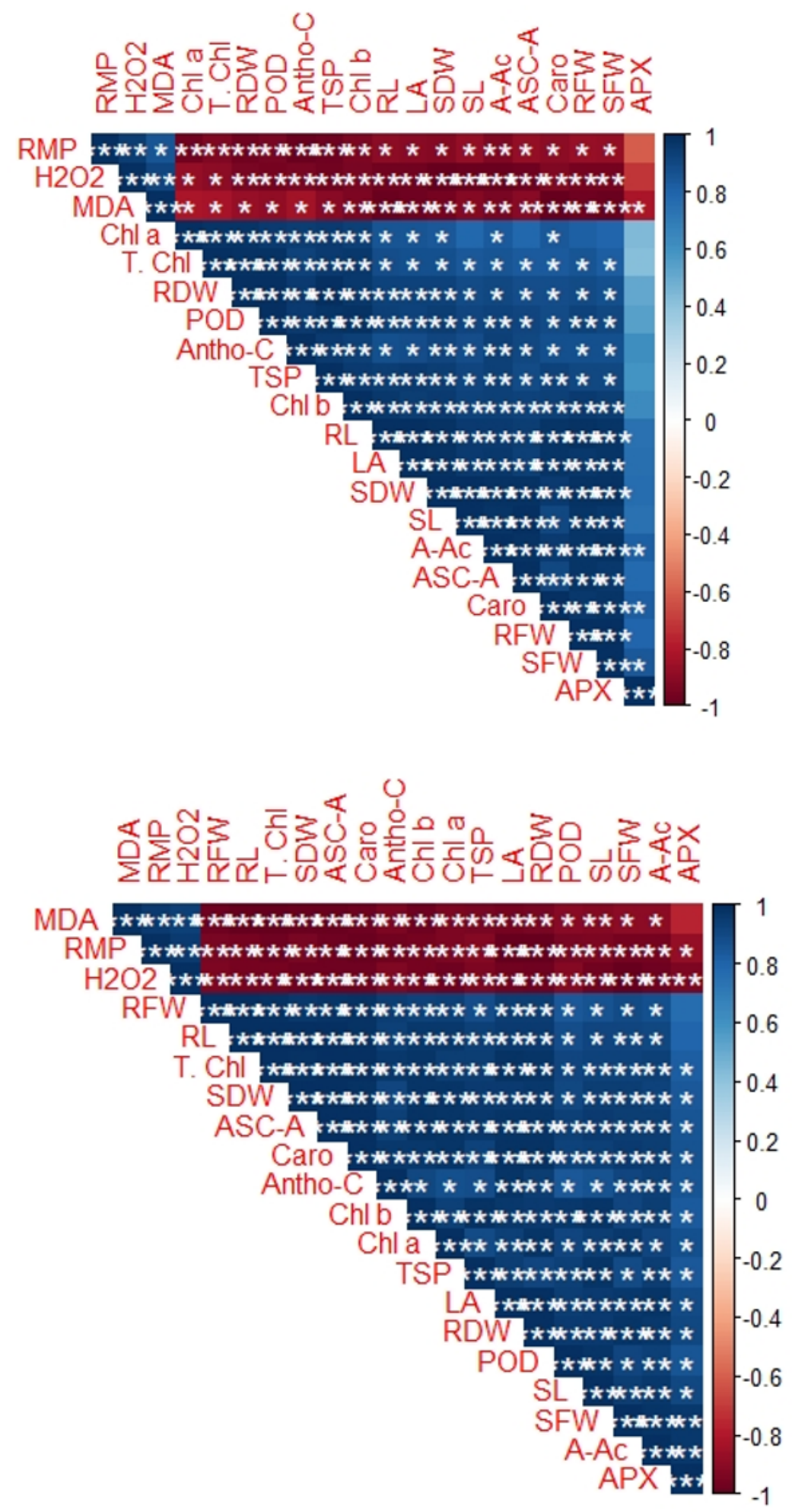

\section{Figure 4}

Correlation among morphological and physiochemical traits of maize seedlings under $(A)$ under control and (B) Cd stress condition. Abbreviations: Melanoaldehyde (MDA); Relative membrane permeability (RMP); Hydrogen per oxide (H2O2); Root fresh weight (RFW); Root length (RL); Total chlorophyll (T.Chl); Shoot dry weight (SDW); Ascorbic acid (ASC-A); Carotenoids (Caro); Anthocyanin contents (Antho-C); 
Chlorophyll b (Chl b); Chlorophyll a (Chl a); Total soluble proteins (TSP); Leaf area (LA); Root dry weight (RDW); Peroxidase (POD); Shoot fresh weight (SFW); Ascorbate per oxidase (APX)

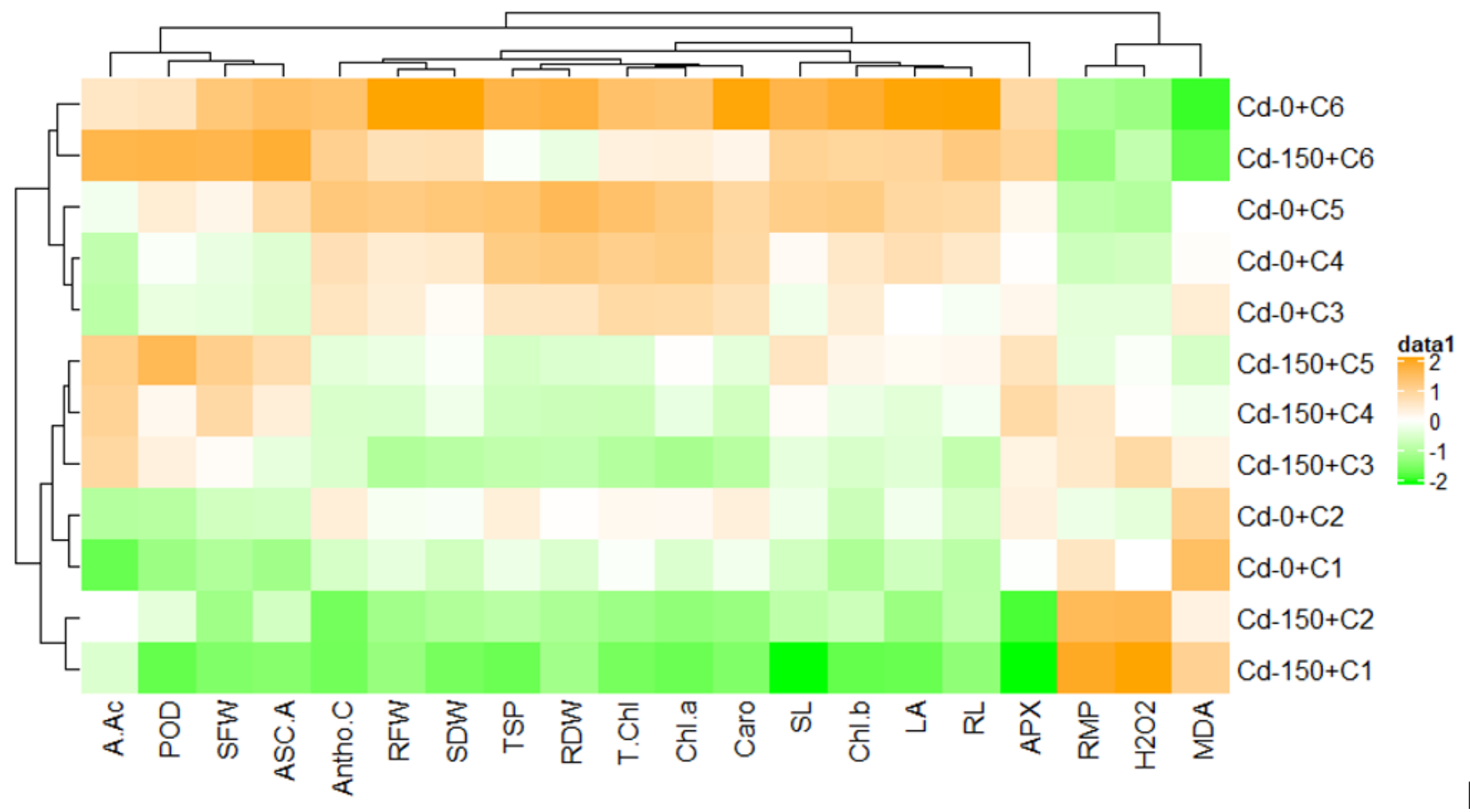

\section{Figure 5}

Clustered heatmap representing the effect of $\mathrm{Cd}$ and $\mathrm{Ca}$ (C1- $0 \mathrm{mM}, \mathrm{C} 2-0.5 \mathrm{mM}, \mathrm{C} 3-1 \mathrm{mM}, \mathrm{C} 4-2.5 \mathrm{mM}$, C5- 5 mM, C6- 10 mM) treatments on different studied traits Figure abbreviations: Melanoaldehyde (MDA); Relative membrane permeability (RMP); Hydrogen per oxide (H2O2); Root fresh weight (RFW); Root length (RL); Total chlorophyll (T.Chl); Shoot dry weight (SDW); Ascorbic acid (ASC-A); Carotenoids (Caro); Anthocyanin contents (Antho-C); Chlorophyll b (Chl b); Chlorophyll a (Chl a); Total soluble proteins (TSP); Leaf area (LA); Root dry weight (RDW); Peroxidase (POD); Shoot fresh weight (SFW); Ascorbate per oxidase (APX) 
(a)

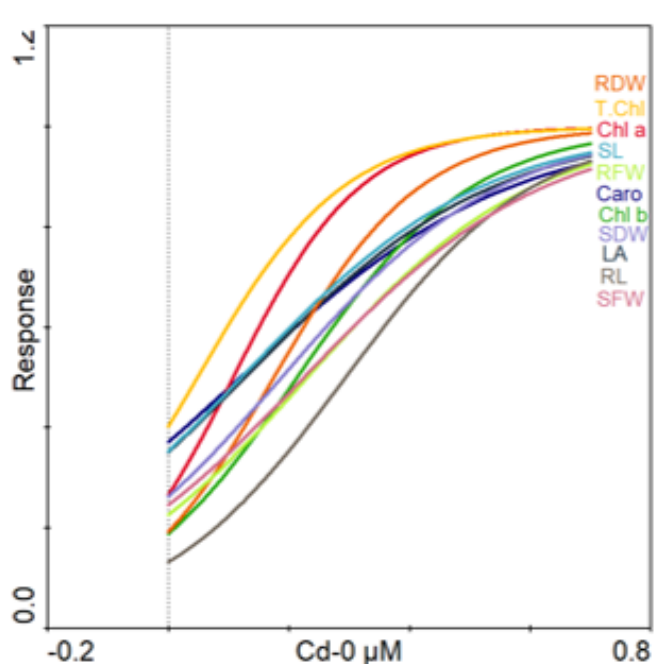

(b)

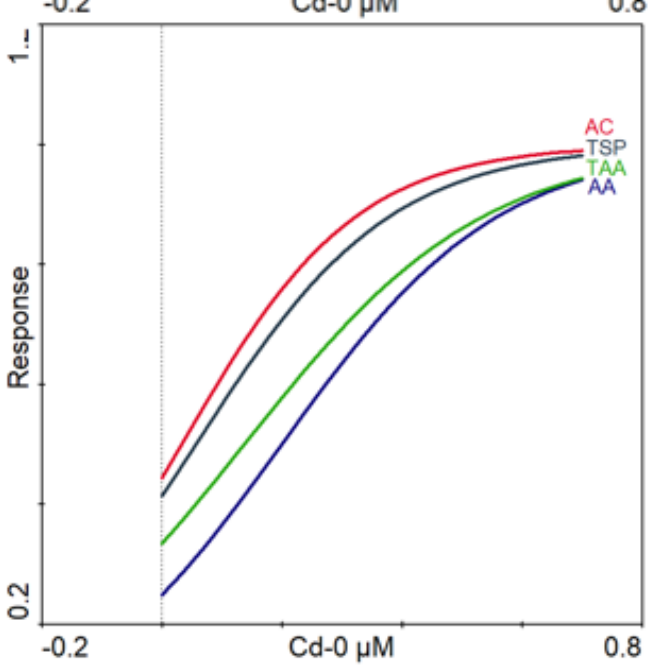

(c)

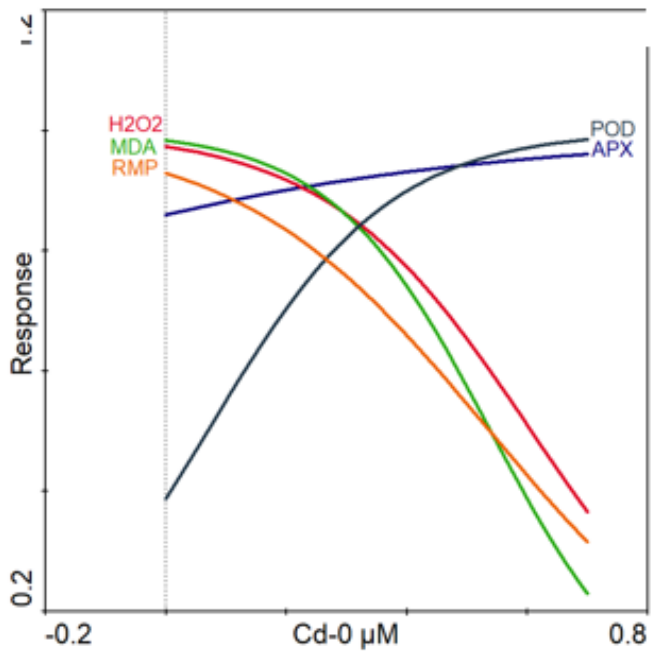

(d)

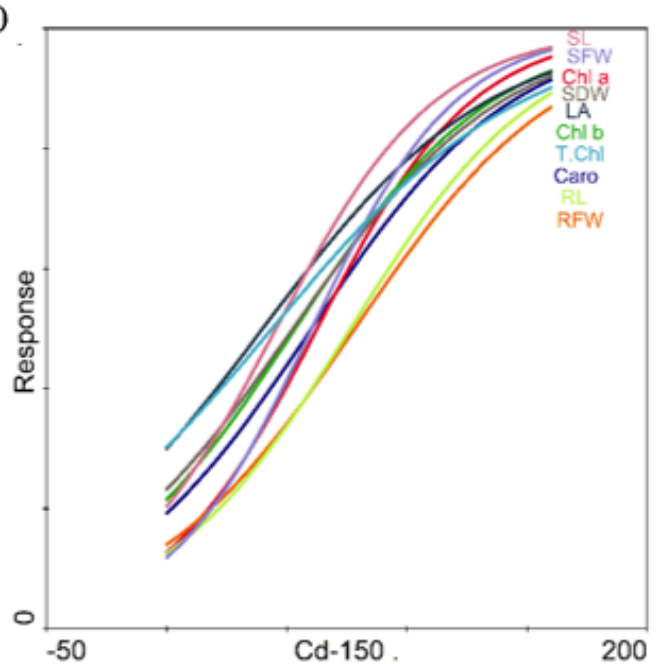

(e)

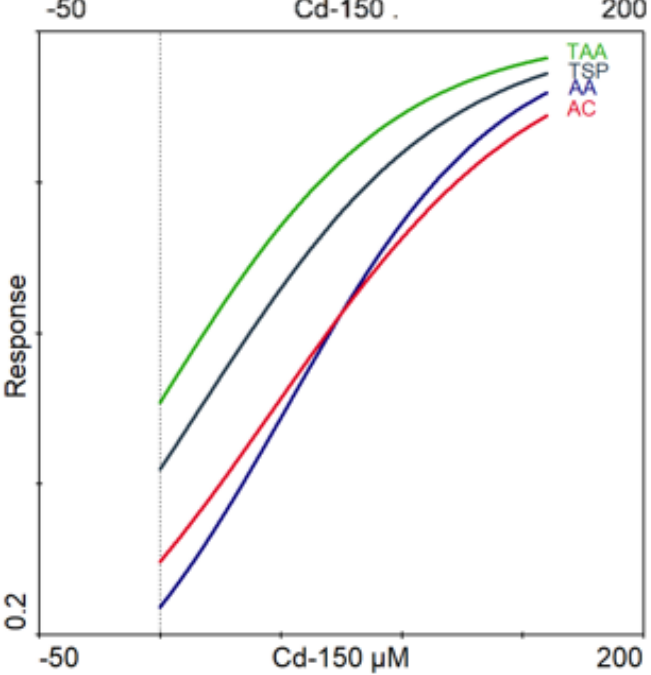

(f)

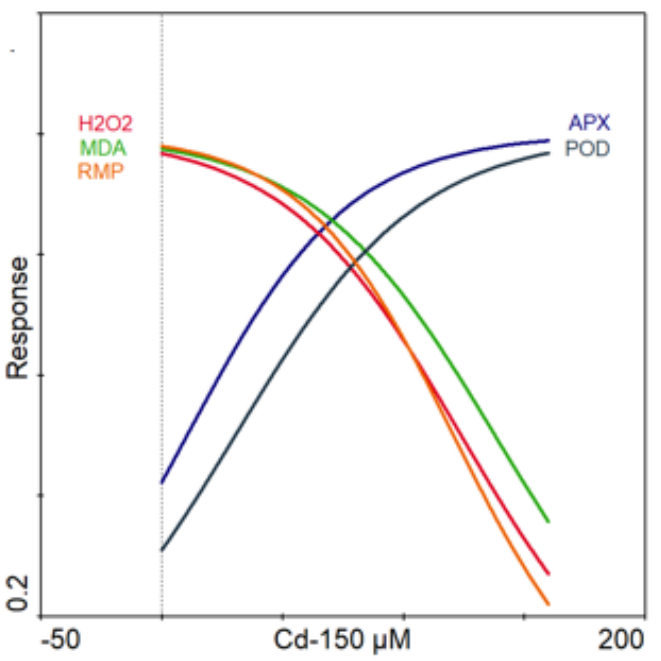

\section{Figure 6}

Generalized linear model showing response curve of traits under $\mathrm{Cd}$ and Ca treatments $\mathrm{Cd}-0 \mu \mathrm{M}$ stress: (a) growth and chlorophyll (b) organic osmolytes, ascorbic acid and anthocynin contents (c) hydrogen peroxide, relative membrane permeability, lipidperoxidation and antioxdants. Cd- $150 \mu \mathrm{M}$ stress; (d) growth and chlorophyll (e) organic osmolytes, ascorbic acid and anthocynin contents (f) hydrogen peroxide, relative membrane permeability, lipidperoxidation and antioxdants Abbreviations: 
Melanoaldehyde (MDA); Relative membrane permeability (RMP); Hydrogen per oxide (H2O2); Root fresh weight (RFW); Root length (RL); Total chlorophyll (T.Chl); Shoot dry weight (SDW); Ascorbic acid (ASC-A); Carotenoids (Caro); Anthocyanin contents (Antho-C); Chlorophyll b (Chl b); Chlorophyll a (Chl a); Total soluble proteins (TSP); Leaf area (LA); Root dry weight (RDW); Peroxidase (POD); Shoot fresh weight (SFW); Ascorbate per oxidase (APX). 